\title{
Simulation Of Optimal Portfolio Using Single Index Model And Markowitz Model On Lq-45 Index Shares For 2018
}

\author{
Novi Puji Lestari ${ }^{1}$, S.E.,M.M $M^{2}$ \\ Fakultas Ekonomi dan Bisnis, Universitas Muhammadiyah Malang, Indonesia
}

The essence of portfolio formation is to reduce risk by means of diversification, namely allocating a number of funds to various investment alternatives that are negatively correlated. To select returns in a portfolio, you can use the Single Index Model and the Markowitz Model. This study was conducted with the aim of comparing the calculation of which company portfolios can provide a good rate of return with a small risk using the Single Index and Markowitz Model based on the sector of the company. So that the results of this study can provide recommendations to investors in decision making on portfolio selection. The research was conducted on companies indexed by LQ 45 on the Indonesia Stock Exchange. The research period is to use companies indexed by LQ 45 in 2018 for two periods.

Keyword: Simulation,Single Indeks Model,LQ 45,Markowitz

OPEN ACCESS

ISSN 2528-4649 (online) ISSN 2338-4409 (print)

Reviewed by: Dr. Eko Purwanto, SE, Msi

${ }^{*}$ Correspondence: Novi Puji Lestari novipujilestari61@gmail.com Received: December 30, 2020

Accepted: February 22, 2021 diterima Published: March 31, 2021 JBMP: Jurnal Bisnis, Manajemen dan Perbankan Vol: $7 /$ No. 1 doi: 10.21070/jbmp.v7i1.880 


\section{INTRODUCTION}

The Capital Market is a market that brings together parties that offer and require long-term funds, both debt securities (bonds), equity (stocks), mutual funds, derivative instruments and other instruments. The capital market is a means of funding for companies and other institutions and as a means of investing activities. The capital market facilitates various facilities and infrastructure for buying and selling long-term instruments and other related activities. The capital market is a place for trading securities that has been carried out in an organized manner. A system structured to bring together sellers and buyers of securities, through intermediaries and direct investors.

Stock Exchange is an institution that provides system facilities to bring together sellers and buyers of long-term securities between various companies with the aim of trading company securities that have been listed on the stock exchange. The number of companies currently listed on the stock exchange is 562 companies. Securities companies or often referred to as securities companies are parties that conduct business as underwriters, securities brokers or investment managers. In the Indonesian Stock Exchange, there are currently 108 securities companies that are members and carry out their activities, both as underwriters, securities brokers and investment managers. The existence of the capital market is very helpful for economic players in finding alternative funding for business activities and investors who want to invest their funds. One of them is stock investment in companies that have been listed on the Indonesia Stock Exchange.

When a person decides to invest in financial assets such as stocks, he expects that in the future, the benefits obtained from investing activities are referred to as returns. The expected return on investment is a compensation for the opportunity cost and the risk of decreasing purchasing power due to the influence of inflation (Tandelilin, 2001). In the context of investment, returns are the returns obtained from investment returns. Returns are divided into two, namely the yield that has occurred which is calculated based on historical data and the two returns that are expected to be obtained by investors in the future. The expected returns are simply the weighted average of various historical returns. The weighing factor is the probability of each return, while the portfolio return is the weighted average of the expected single share returns (Halim, 2005).

Errors in stock selection will affect the provision of returns, so that the returns obtained from the portfolio are not as expected. To obtain the desired portfolio, investors must carry out an analysis that provides the maximum return. A rational investor will choose a portfolio that will provide the maximum return on a certain risk. A portfolio is a combination or combination or set of assets, both in the form of real assets and financial assets owned by investors. (Tandelilin, 2010) The essence of portfolio formation is to reduce risk by means of diversification, namely allocating a number of funds to various investment alternatives that are negatively correlated. To select returns in a portfolio, you can use a model Single Index and Markowitz Model.
The index model or factor model assumes that a security's yield is sensitive to changes in a variety of factors. The index model seeks to include the major economic forces that can systematically move the prices of all securities. The single index model or the one-factor model assumes that the yields between two or more securities will be correlated i.e. they will move together and have the same reaction to one factor, namely the JCI Composite Stock Price Index). (Halim, 2018)

Markowitz or multi-index models have more potential in an effort to estimate expected return, standard deviation and effect covariance more accurately than single index models. Because the actual return of securities is not only sensitive to changes in the JCI, it means that there is a possibility that more than one factor can influence it. Multi index models assume that there are other factors besides the IHSG that can influence the correlation between effects, for example the risk-free interest rate (Halim, 2005).

The LQ45 Stock Index is a stock market index on the Indonesia Stock Exchange (IDX) consisting of 45 issuers with the largest market capitalization and the highest transaction value on the regular market in the last 12 months. Issuers can only be listed on LQ45 shares if they have been listed on the IDX for at least three months, and have high financial conditions and growth prospects. Therefore, the issuers in the LQ45 stock list are often referred to as superior stocks or favorite stocks. (https://www.seputarforex.com/saham/lq_45) 1 .

\section{METHOD (FOR RESEARCH ARTICLE)}

\section{TYPES OF RESEARCH}

This research is a descriptive study, namely "Research conducted to determine the independent variables, either one or more (independent) variables without making comparisons, or connecting with other variables". Descriptive research is "Research that is directed to provide symptoms, facts, or events in a systematic and accurate manner, regarding the characteristics of a particular population or area".

The approach used in this study is a quantitative approach, because the main data used in this study are numeric data. "Quantitative data is data in the form of numbers, or qualitative data that is extrapolated." This research uses data in the form of a list of monthly stock closing prices, monthly Composite Stock Price Index (IHSG) and BI interest rates for the period 2018. The data will be processed or processed using formulas from several theories which will then be analyzed and described so that it can a conclusion is reached.

\section{POPULATION AND SAMPLES}

The population in this study were all companies included in the LQ-45 index for the 2018 period, namely 45 companies. From 45 companies, it will be seen whether they are active in stock trading in the last 1 year or not. Then we will classify the calculations based on the company's industrial sector.

\section{TYPES AND DATA SOURCES}

The data collected is in the form of secondary data obtained from the Indonesia Stock Exchange Corner and from the Bank Indonesia website during 2018. Secondary data is the source of 
research data obtained through intermediary media or indirectly in the form of books, records, existing evidence, or archives. both published and not publicly published. The data required is the closing price of shares every month, the BI Rate and the Composite Stock Price Index each month during 2018.

\section{VARIABLES}

An operational definition is a definition based on the observable characteristics of what is being defined or changing the concepts that describe observable behaviors or symptoms that can be tested and verified. Some of the indicators used in assessing the optimal portfolio are as follows:

Actual Return is a historical return that has occurred and can also be called a realize return. To get stock returns, many methods are used, such as probabilty returns and nonprobability returns and dividends.

Expected Return is the rate of return expected by an investor Shares Variance is a measurement of the risk of return expected by investors on certain stocks.

Alpha is a linear relationship between actual stock returns and market actual returns (IHSG). This alpha is used to calculate Variance Error (ei).

Beta is the slope of the linear relationship between actual stock returns. Beta is also called systematic risk or risk that cannot be diversified through portfolio formation.

Variance Error Residual, namely the unsystematic risk of a stock

Cut Off Rate calculates which stocks can provide optimal value.

\section{DATA ANALYSIS}

The data analysis technique in this study uses the Single Index model analysis, which is able to calculate manually or calculate using Ms. Excel in calculating the optimal portfolio of stocks. (Prasetyo, 2017) The data analysis technique carried out is as follows:

Single Index Model

Calculating the return on the realization of each share by calculating the dividend per montH

$R_{i, t}=\frac{P_{t}-P_{t-1}}{P_{t-1}}$

Calculating Expected Return

$$
\mathrm{E}(\mathrm{R})=\sum \frac{R i}{n}
$$

Calculating Stock Variance

Variants can be calculated by the following formula:

$\sigma i^{2}=\sum_{n-1}^{n}\left(R i-\frac{E(R i) 2}{n-1}\right.$

Calculating Alpha

Alpha is used to calculate the error variance.

Manually alpha can be calculated as follows:
$\propto_{i}=R_{i}-\beta_{i} x R_{m}$

Calculating Stock Beta

Beta can be manually calculated using the following formula:

$\beta_{i}=\frac{\sigma i m}{\sigma m}$

Calculating Stock Residual Variance Error

$$
e_{i}=R_{i}-\propto_{i}-\left(\beta_{i} x R_{m}\right)
$$

Calculating Excess Return

Excess Return $\quad=R_{i}-R_{f}$

Calculating Excess Return to Beta

$\mathrm{ERB}=E\left(R_{i)}-\frac{R f}{\beta i}\right.$

Sort of Excess Return to Beta

Calculating the Value of $\mathrm{Ai}$ and $\mathrm{Bi}$ for each of the ith securities as follows:

$\mathrm{Ai}=\frac{E(R i)-R(B r) \cdot \beta i}{\sigma e i}$

$\mathrm{Bi}=\frac{\beta i}{\sigma e i}$

Calculating the size of the proportion for each security after the securities that make up the optimal portfolio can be determined

$\mathrm{Wi}=\frac{Z i}{\sum Z j}$

\section{Markowitz indeks Model}

By using a simple linear regression equation will be used to calculate the factors that are thought to affect stock prices. (Eka Pratiwi, Dzulkirom, \& Farah Azizah, 2014). This Markowitz Index Model can be done in several steps, namely:

Calculate the return on individual stock realization

Calculate the expected return on individual stocks

Calculating Standard Deviation and Variance

Calculating Covarian

Calculating the Correlation Coefficient

Calculating the Portfolio's Expected Return

Calculating the Standard Deviation of the Portfolio

\section{RESULTS AND DISCUSSION}

\section{Gambaran Umum Perusahaan}

Setelah dilakukan pengamatan maka sampel yang digunakan dalam penelitian ini berjumlah 37 perusahaan yang aktif melakukan perdagangan saham dalam 1 tahun terakhir. Data perusahaan tersebut dirangkum dalam Tabel 4.1 berikut:

[Table 4.1 about here.] 


\section{Tahap Perhitungan Model Single Indeks}

Tabulasi Data diperlukan untuk mempermudah peneliti dalam mengolah data penelitian ini,Adapun data yang diolah dalam bentuk harga penutupan saham ,Indeks Harga Saham Gabungan dan Suku Bunga Bank Indonesia terangkum dalam tabel-tabel di bawah ini :

[Table 4.2 about here.]

[Table 4.3 about here.]

[Table 4.6 about here.]

[Table 4.7 about here.]

[Table 4.8 about here.]

[Table 4.9 about here.]

\section{Actual Return}

Actual Return is a historical return that has occurred and can also be called a realize return. The formula for calculating Actual Return is as follows:

$R_{i, t}=\frac{P_{t}-P_{t-1}}{P_{t-1}}$
[Table 5.3 about here.]

[Table 5.4 about here.]

[Table 5.5 about here.]

[Table 5.6 about here.]

[Table 5.7 about here.]

Calculation of Expected Return, Stock and Market Variance, Calculating Stock Alpha and Beta, Calculating Variance Error

[Table 5.8 about here.]

[Table 5.9 about here.]

[Table 5.11 about here.]

[Table 5.12 about here.]

[Table 5.13 about here.]

[Table 5.14 about here.]

[Table 5.0 about here.]

[Table 5.15 about here.]

[Table 5.1 about here.]

[Table 5.2 about here.]

Calculation of Excess Return, ERB, Calculating Cut of Rate, Sorting Shares based on ERB, Determining Cut off 


\section{Rate and Determining Shares which are included in the Optimal Portfolio}

[Table 5.16 about here.]

[Table 5.17 about here.]

[Table 5.18 about here.]

[Table 5.19 about here.]

[Table 5.20 about here.]

[Table 5.21 about here.]

[Table 5.22 about here.]

[Table 5.23 about here.]

From the results of calculations using the Single Index Model above, it is found that the optimal portfolio component is for property companies, all companies are optimal, this is evidenced by the calculation of Excess Return to Beta is greater than the Cut of Point value of the optimal portfolio combination, then for mining companies there is 2 that are optimal, namely INCO and PTBA, there is only one trade company that is optimal, namely UNTR, almost all of its share components are optimal except for Bank Mandiri, for Misc Ind. Companies there are SRIL and Basic Ind. BRPT, SMGR, INKP. Infrastructure companies are JSMR, PGAS and TLKM and the last combination is the consumer sector, there is only 1 company, namely KLBF.

It can be concluded that in the calculation of the optimal portfolio calculation simulation using the Single Model Index, the best taken by consumers is the property company because all companies have a very good level of return and risk in the preparation of optimal portfolios. If consumers want to combine various types of portfolios, they can combine portfolios in property companies with finance companies, or it can be with Basic Ind and Infrastructure companies, all of which provide high returns for investors.

\section{Markowitz Model}

The Markowitz model is a method that can be used as an alternative by investors as a basis for making investment decisions. The basic components in calculations using the Markowitz Model are less than calculations using the Single Index Model. The following is the calculation of stock composition using the Markowitz model.

[Table 5.24 about here.]

[Table 5.25 about here.]

[Table 5.26 about here.]

[Table 5.27 about here.]

[Table 5.28 about here.]

[Table 5.29 about here.]

[Table 5.30 about here.]

[Table 5.31 about here.]

From calculations using the Markowitz model, it can be seen that the company that has the highest realization return of all companies is in the Mining Company, namely Aneka Tambang (ANTM) and the Basic Ind. Company, namely Indocement Tunggal Perkasa (INTP). Portfolio in this case does not directly combine one stock with another stock and not only considers the aspects of return and risk, but also considers the covariance and correlation coefficient of these stocks. The correlation coefficient is a calculation that describes the movements between stocks which are combined to determine the effect between stocks.

However, in terms of calculation for the proportion of optimal portfolio-forming stock funds using the Markowitz method, it is known that the stocks that form a high optimal portfolio are property companies by WIKA, Mining Companies 
by ANTM, INCO, PTBA, Trade Companies, all companies are optimal, and the optimal Ind. Misc Sector. is ASII, the optimal finance companies are BBCA, BBRI and BMRI. The optimal Basic Ind companies are BRPT, SMGR, WSBP while the infrastructure company is PGAS and finally the Consumer companies are GGRM, ICBP, INDF and UNVR.

\section{CONCLUSIONS}

Based on the 45 companies that were included in the LQ 45 Index in 2018, it turned out that there were 37 companies that could be included in the criteria for the sample company because in the two periods the company was actively listing on the Indonesia Stock Exchange. According to calculations using the Single Index Model of 37 companies that form an optimal portfolio, there are 20 companies with the highest proportion in the property sector where all companies are optimal, then the finance sector almost all of the companies form an optimal portfolio except for Bank Mandiri, then underneath there is the Infrastructure sector, Basic Industry, Misc Industry, Mining, Trade, and Consumer.

If the calculation uses the Markowitz model, there are 22 companies whose portfolio formation is good. This is clearly different from the two analyzes of the calculations. The optimal portfolio formation from the calculation results of the Markowitz method is the Trade, Finance, Basic-Inc, Mining, Consumer, Property, Misc Ind and Infrastructure sectors. This proves that if the calculation or simulation uses two methods, namely the single index model method and the Markowitz method, the results are different, because in Markowitz theory there are other factors that must be considered in the formation of an optimal portfolio, not only on the Composite Stock Price Index. 


\section{REFERENCES}

Abdul Halim Universitas Gajayana. (2018). Analisis Investasi dan Aplikasinya (2nd ed.). Jakarta: Salemba Empat.

Eka Pratiwi, A., Dzulkirom, M., \& Farah Azizah, D. (2014a). Analisis Investasi Portofolio Saham Pasar Modal Syariah Dengan Model Markowitz Dan Model Indeks Tunggal. Jurnal Administrasi Bisnis, 17(Desember), 1-10.

Eka Pratiwi, A., Dzulkirom, M., \& Farah Azizah, D. (2014b). Analisis Investasi Portofolio Saham Pasar Modal Syariah Dengan Model Markowitz Dan Model Indeks Tunggal. Jurnal Administrasi Bisnis.

Halim, A. (2005). Analisis investasi. Jakarta: Salemba Empat.

Jorgiyanto H.M. (2008). Analisis dan Desain Sistem Informasi: Pendekatan Terstruktur Teori dan Praktek Aplikasi Bisnis. In Andi Publisher.

Kertopati, B. (2013). Analisa Pembentukan Portofolio Dengan Menggunakan Model Markowitz Dan Single Index Model pada Saham yang masuk Dalam Indeks LQ45 Di Bursa Efek Indonesia Tahun 2009 - 2013.

Prasetyo, A. T. (2017). Analisis Pembentukan Portofolio Optimal Dengan Menggunakan Model Indeks Tunggal Pada Indeks Saham LQ 45 Periode 2015 - 2016. Jurnal Simki-Economic.

Septyanto, D., \& Kertopati, B. (2014). Analisa Pembentukan Portofolio Dengan Menggunakan Model Markowitz dan Single Index Model Pada Saham Yang Masuk Dalam Indeks LQ45 Di Bursa Efek Indonesia Tahun 2009-2013. Finance and Banking Journal.

Tandelilin, E. (2001). Analisis Investasi dan Manajemen Portofolio. In Analisis Investasi dan Manajemen Portofolio11.

Tandelilin, E. (2010). Portofolio dan Investasi Teori dan Aplikasi. In Yogyakarta: Kanisius. https://doi.org/10.2753/MIS07421222260306

Conflict of InterestStatement:Theauthorsdeclare that theresearch wasconducted in the absence of any commercial or financial relationships that could be construed as a potential conflict of interest.

Copyright (C) tahun terbit nama belakang and nama belakang. This is an open-access article distributed under the terms of the Creative Commons Attribution License (CC $B Y)$. The use, distribution or reproduction in other forums is permitted, provided the original author(s) and the copyright owner(s) are credited and that the original publication in this journal is cited, in accordance with accepted academic practice. No use, distribution or reproduction is permitted which does not comply with these terms. 


\section{LIST OF TABLES}

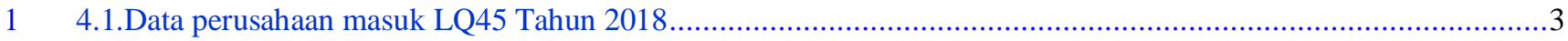

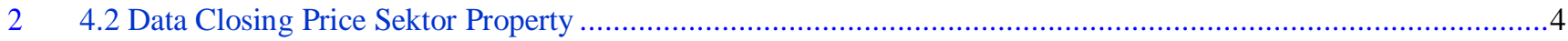

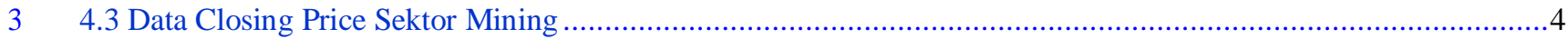

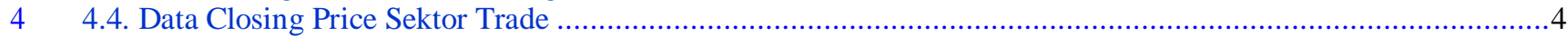

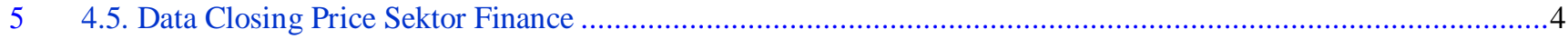

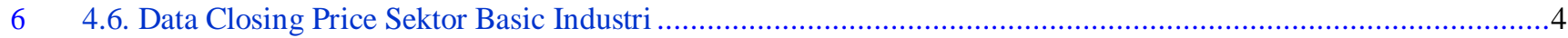

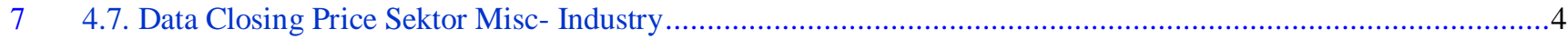

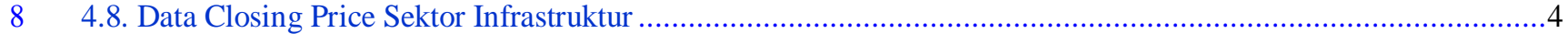

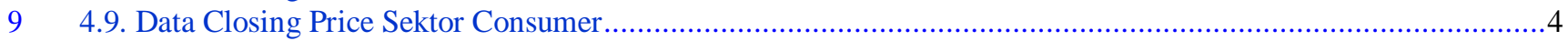

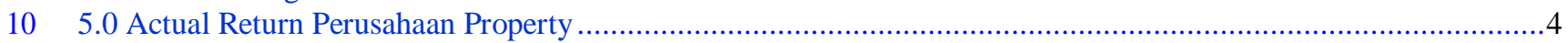

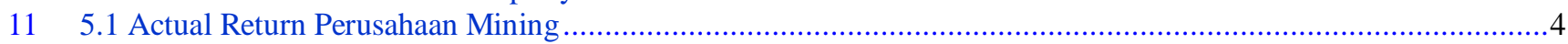

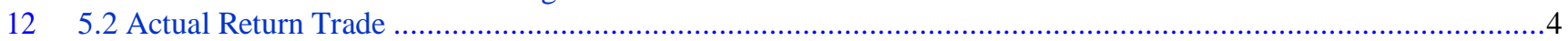

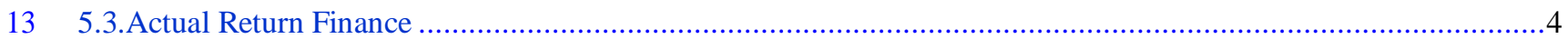

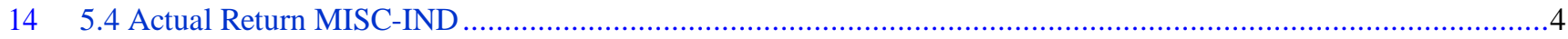

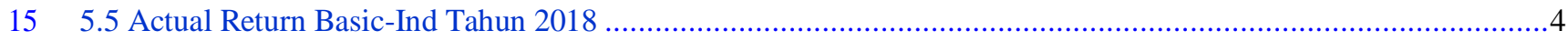

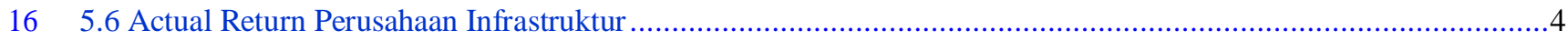

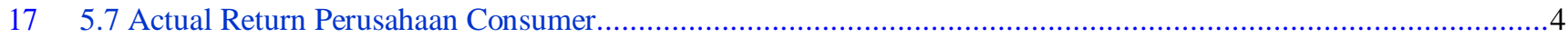

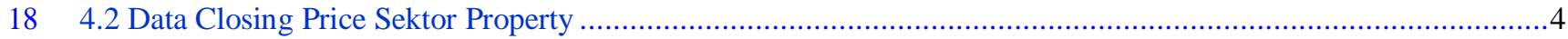

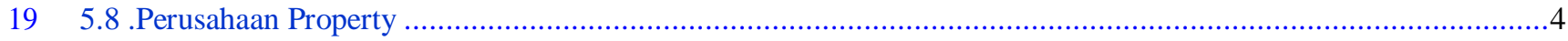

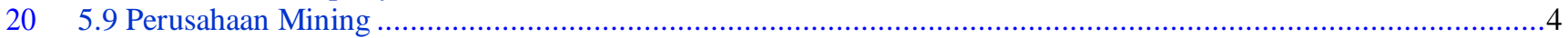

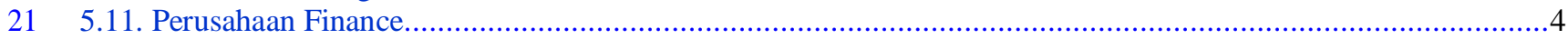

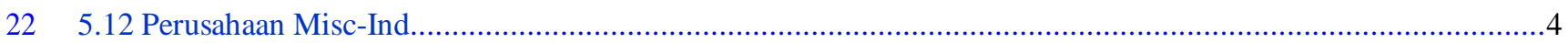

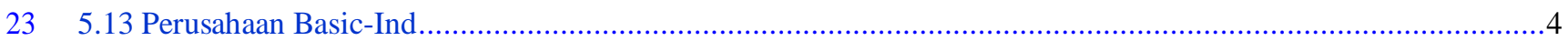

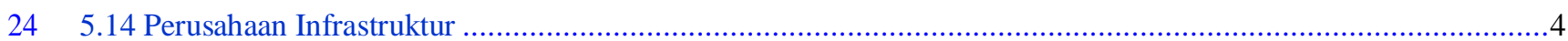

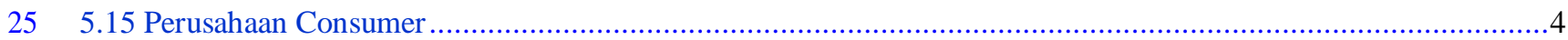

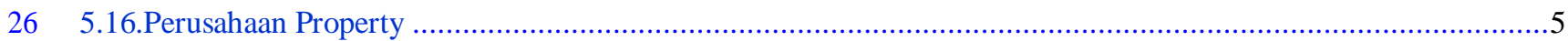

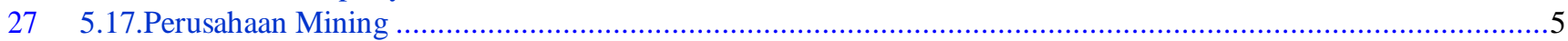

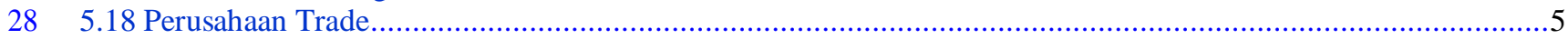

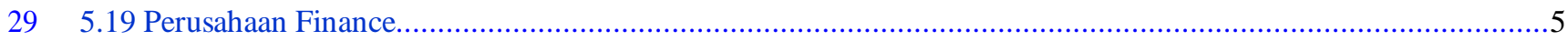

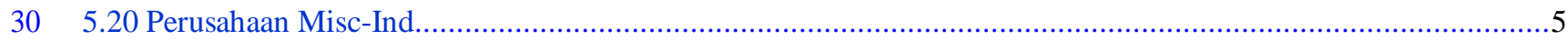

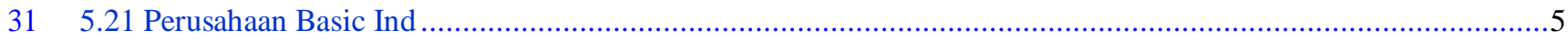

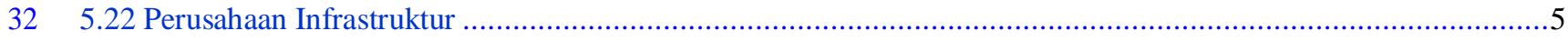

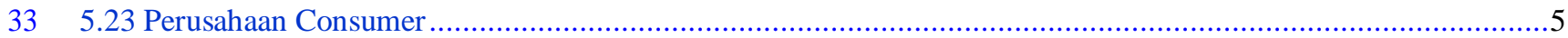

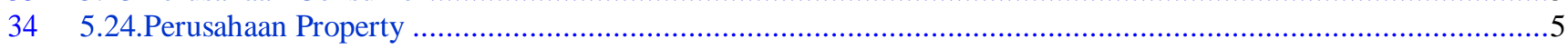

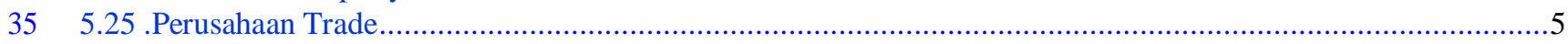

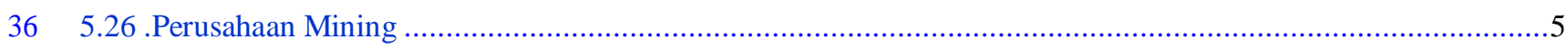

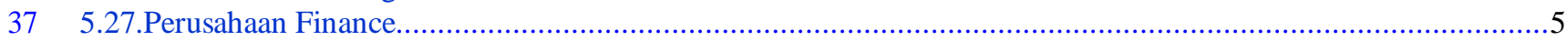

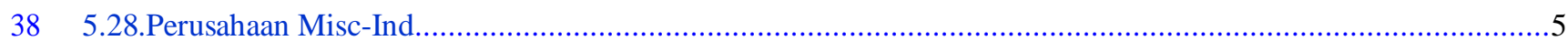

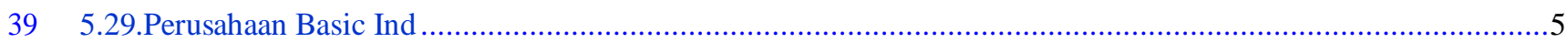

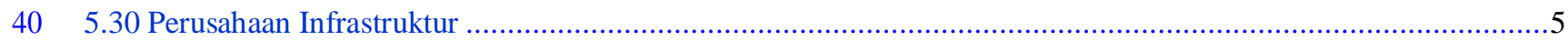

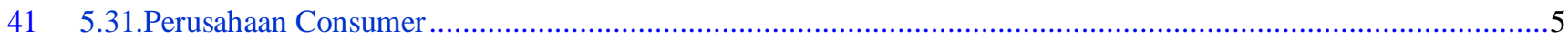


TABLE 4.1 / Data perusahaan masuk LQ45 Tahun 2018

\begin{tabular}{|c|c|c|}
\hline No & Sektor & Nama Saham \\
\hline 1. & Property & $\begin{array}{ll}\text { 1. } & \text { ADHI } \\
\text { 2. } & \text { BSDE } \\
\text { 3. } & \text { PT.PP } \\
\text { 4. } & \text { WIKA } \\
\text { 5. } & \text { WSKT }\end{array}$ \\
\hline 2. & Mining & $\begin{array}{ll}\text { 1. } & \text { ADRO } \\
\text { 2. } & \text { ANTM } \\
\text { 3. } & \text { INCO } \\
\text { 4. } & \text { PTBA }\end{array}$ \\
\hline 3. & Trade & $\begin{array}{ll}\text { 1. } & \text { AKRA } \\
\text { 2. } & \text { LPPF } \\
\text { 3. } & \text { MNCN } \\
\text { 4. } & \text { SCMA } \\
\text { 5. } & \text { UNTR } \\
\end{array}$ \\
\hline 4. & Misc Ind & $\begin{array}{ll}\text { 1. } & \text { ASII } \\
\text { 2. } & \text { SRIL }\end{array}$ \\
\hline 5. & Finance & $\begin{array}{ll}\text { 1. } & \text { BBCA } \\
\text { 2. } & \text { BBNI } \\
\text { 3. } & \text { BBRI } \\
\text { 4. } & \text { BBTN } \\
\text { 5. } & \text { BMRI } \\
\end{array}$ \\
\hline 6. & Basic Ind & $\begin{array}{ll}\text { 1. } & \text { BRPT } \\
\text { 2. } & \text { INTP } \\
\text { 3. } & \text { SMGR } \\
\text { 4. } & \text { WSBP } \\
\text { 5. } & \text { TPIA } \\
\end{array}$ \\
\hline 7. & Infrastruktur & $\begin{array}{ll}\text { 1. } & \text { EXCL } \\
\text { 2. } & \text { INDY } \\
\text { 3. } & \text { JSMR } \\
\text { 4. } & \text { PGAS } \\
\text { 5. } & \text { TLKM } \\
\end{array}$ \\
\hline 8. & Consumer & $\begin{array}{ll}\text { 1. } & \text { GGRM } \\
\text { 2. } & \text { HMSP } \\
\text { 3. } & \text { ICBP } \\
\text { 4. } & \text { INDF } \\
\text { 5. } & \text { KLBF } \\
\text { 6. } & \text { UNVR }\end{array}$ \\
\hline
\end{tabular}

Sumber : www.idx.go.id 
TABLE 4.2 / Data Closing Price Sektor Property

\begin{tabular}{|c|c|c|c|c|c|c|l|}
\hline Bulan & \multicolumn{5}{|c|}{ Close Price } & & \\
\hline & \multicolumn{5}{|c|}{ PROPERTY } & \\
\hline & ADHI & BSDE & PT.PP & WIKA & WSKT & IHSG & RF \\
\hline Jan-18 & 2230 & 1820 & 3130 & 2080 & 2830 & 6650 & $4.25 \%$ \\
\hline Feb-18 & 2480 & 1935 & 3100 & 1925 & 2910 & 6600 & $4.25 \%$ \\
\hline Mar-18 & 2070 & 1780 & 2610 & 1680 & 2470 & 6690 & $4.25 \%$ \\
\hline Apr-18 & 1880 & 1690 & 2430 & 1585 & 2210 & 5650 & $4.50 \%$ \\
\hline Mei-18 & 1945 & 1705 & 2580 & 1670 & 2320 & 5650 & $4.75 \%$ \\
\hline Jun-18 & 1790 & 1565 & 1995 & 1325 & 1925 & 5620 & $5.25 \%$ \\
\hline Jul-18 & 1605 & 1350 & 2080 & 1550 & 2120 & 5800 & $5.25 \%$ \\
\hline Agu-18 & 1490 & 1200 & 1900 & 1550 & 1895 & 5900 & $5.50 \%$ \\
\hline Sep-18 & 1390 & 1155 & 1525 & 1365 & 1700 & 5650 & $5.75 \%$ \\
\hline Okt-18 & 1125 & 1100 & 1330 & 1100 & 1440 & 5650 & $5.75 \%$ \\
\hline Nov-18 & 1565 & 1350 & 1855 & 1505 & 1560 & 6150 & $6.00 \%$ \\
\hline Des-18 & 1585 & 1255 & 1805 & 1655 & 1680 & 6600 & $6.00 \%$ \\
\hline Jan-19 & 1630 & 1330 & 2340 & 1895 & 1975 & 6650 & $6.00 \%$ \\
\hline
\end{tabular}


TABLE 4.3 / Data Closing Price Sektor Mining

\begin{tabular}{|r|r|r|r|r|r|l|}
\hline Bulan & \multicolumn{5}{|c|}{ Close Price } \\
\hline & \multicolumn{5}{|c|}{ MINING } \\
\hline & ADRO & ANTM & INCO & PTBA & IHSG & RF \\
\hline Jan-18 & 2450 & 915 & 3750 & 3400 & 6650 & $4.25 \%$ \\
\hline Feb-18 & 2350 & 955 & 3390 & 3170 & 6600 & $4.25 \%$ \\
\hline Mar-18 & 2130 & 775 & 2790 & 2940 & 6690 & $4.25 \%$ \\
\hline Apr-18 & 1835 & 845 & 3160 & 3240 & 5650 & $4.50 \%$ \\
\hline Mei-18 & 1885 & 865 & 3860 & 3800 & 5650 & $4.75 \%$ \\
\hline Jun-18 & 1790 & 890 & 4040 & 3970 & 5620 & $5.25 \%$ \\
\hline Jul-18 & 1905 & 915 & 4370 & 4480 & 5800 & $5.25 \%$ \\
\hline Agu-18 & 1865 & 5220 & 3800 & 4050 & 5900 & $5.50 \%$ \\
\hline Sep-18 & 1835 & 5070 & 3710 & 4320 & 5650 & $5.75 \%$ \\
\hline Okt-18 & 1650 & 4080 & 2920 & 4250 & 5650 & $5.75 \%$ \\
\hline Nov-18 & 1285 & 3690 & 3030 & 4020 & 6150 & $6.00 \%$ \\
\hline Des-18 & 1215 & 4590 & 3260 & 4300 & 6600 & $6.00 \%$ \\
\hline Jan-19 & 1390 & 1930 & 3850 & 4310 & 6650 & $6.00 \%$ \\
\hline
\end{tabular}


TABLE 4.4 / Data Closing Price Sektor Trade

\begin{tabular}{|l|l|l|l|l|l|l|l|}
\hline Bulan & \multicolumn{5}{|c|}{ Close Price } & & \\
\hline & \multicolumn{5}{|c|}{ TRADE } & \\
\hline & AKRA & LPPF & MNCN & SCMA & UNTR & IHSG & RF \\
\hline Jan18 & 6225 & 11125 & 1525 & 710 & 38900 & 6650 & $4.25 \%$ \\
\hline Feb18 & 6100 & 10650 & 1535 & 675 & 35600 & 6600 & $4.25 \%$ \\
\hline Mar18 & 5675 & 10950 & 1415 & 630 & 32000 & 6690 & $4.25 \%$ \\
\hline Apr18 & 4900 & 10350 & 1325 & 590 & 34100 & 5650 & $4.50 \%$ \\
\hline Mei18 & 4920 & 9150 & 1200 & 560 & 35050 & 5650 & $4.75 \%$ \\
\hline Jun18 & 4300 & 8800 & 920 & 530 & 31600 & 5620 & $5.25 \%$ \\
\hline Jul18 & 4210 & 8025 & 985 & 515 & 35250 & 5800 & $5.25 \%$ \\
\hline Agu18 & 3610 & 7500 & 905 & 515 & 34400 & 5900 & $5.50 \%$ \\
\hline Sep18 & 3670 & 6925 & 805 & 515 & 33000 & 5650 & $5.75 \%$ \\
\hline Okt18 & 3460 & 4850 & 780 & 478 & 33500 & 5650 & $5.75 \%$ \\
\hline Nov18 & 3900 & 4750 & 790 & 590 & 27500 & 6150 & $6.00 \%$ \\
\hline Des18 & 4290 & 5600 & 690 & 620 & 27350 & 6600 & $6.00 \%$ \\
\hline Jan19 & 5250 & 7000 & 845 & 650 & 25725 & 6650 & $6.00 \%$ \\
\hline
\end{tabular}


TABLE 4.5 / Data Closing Price Sektor Finance

\begin{tabular}{|r|c|c|c|c|c|c|l|}
\hline Bulan & \multicolumn{7}{|c|}{ Close Price } \\
\hline & \multicolumn{7}{|c|}{ FINANCE } \\
\hline & BBCA & BBNI & BBRI & BBTN & BMRI & IHSG & RF \\
\hline Jan18 & 22725 & 9400 & 3700 & 3660 & 8150 & 6650 & $4.25 \%$ \\
\hline Feb18 & 23175 & 9725 & 3780 & 3740 & 8300 & 6600 & $4.25 \%$ \\
\hline Mar18 & 23300 & 8675 & 3600 & 3800 & 7675 & 6690 & $4.25 \%$ \\
\hline Apr18 & 22100 & 8050 & 3220 & 3110 & 7125 & 5650 & $4.50 \%$ \\
\hline Mei18 & 22700 & 8475 & 3080 & 3050 & 7050 & 5650 & $4.75 \%$ \\
\hline Jun18 & 21475 & 7050 & 2840 & 2450 & 6850 & 5620 & $5.25 \%$ \\
\hline Jul18 & 23275 & 7400 & 3070 & 2360 & 6650 & 5800 & $5.25 \%$ \\
\hline Agu18 & 24800 & 7800 & 3180 & 2750 & 6900 & 5900 & $5.50 \%$ \\
\hline Sep18 & 24150 & 7400 & 3150 & 2630 & 6725 & 5650 & $5.75 \%$ \\
\hline Okt18 & 23650 & 7325 & 3150 & 2120 & 6850 & 5650 & $5.75 \%$ \\
\hline Nov18 & 26050 & 8500 & 3620 & 2670 & 7400 & 6150 & $6.00 \%$ \\
\hline Des18 & 26000 & 8800 & 3660 & 2540 & 7375 & 6600 & $6.00 \%$ \\
\hline Jan19 & 28175 & 9075 & 3850 & 2740 & 7450 & 6650 & $6.00 \%$ \\
\hline
\end{tabular}


TABLE 4.6 / Data Closing Price Sektor Basic Industri

\begin{tabular}{|l|r|r|r|r|r|r|r|l|}
\hline Bulan & \multicolumn{7}{|c|}{ Close Price } & \\
\hline & \multicolumn{7}{|c|}{ BASIC-IND } \\
\hline & BRPT & INTP & SMGR & VSBP & INKP & TPIA & IHSG & RF \\
\hline Jan18 & 2640 & 4470 & 11150 & 474 & 9275 & 4525 & 6650 & $4.25 \%$ \\
\hline Feb18 & 2620 & 4320 & 11125 & 480 & 10750 & 4150 & 6600 & $4.25 \%$ \\
\hline Mar18 & 2310 & 3530 & 10350 & 414 & 11000 & 4225 & 6690 & $4.25 \%$ \\
\hline Apr18 & 2470 & 3410 & 9650 & 404 & 12200 & 4050 & 5650 & $4.50 \%$ \\
\hline Mei18 & 2120 & 3740 & 8400 & 402 & 14700 & 5850 & 5650 & $4.75 \%$ \\
\hline Jun18 & 1980 & 3420 & 7125 & 366 & 18800 & 5800 & 5620 & $5.25 \%$ \\
\hline Jul18 & 1815 & 3600 & 7600 & 428 & 19200 & 5000 & 5800 & $5.25 \%$ \\
\hline Agu18 & 1715 & 3270 & 9450 & 390 & 19100 & 5500 & 5900 & $5.50 \%$ \\
\hline Sep18 & 1840 & 2800 & 9925 & 358 & 17250 & 4920 & 5650 & $5.75 \%$ \\
\hline Okt18 & 1875 & 2240 & 9000 & 318 & 12725 & 4510 & 5650 & $5.75 \%$ \\
\hline Nov18 & 2110 & 1800 & 12025 & 350 & 10800 & 5225 & 6150 & $6.00 \%$ \\
\hline Des18 & 2390 & 1585 & 11500 & 376 & 11350 & 5225 & 6600 & $6.00 \%$ \\
\hline Jan19 & 2610 & 2130 & 12675 & 384 & 12000 & 5575 & 6650 & $6.00 \%$ \\
\hline
\end{tabular}


TABLE 4.7 / Data Closing Price Sektor Misc- Industry

\begin{tabular}{|r|r|r|r|l|}
\hline Bulan & \multicolumn{4}{|c|}{ Close Price } \\
\hline & \multicolumn{4}{|c|}{ MISC - IND } \\
\hline & \multicolumn{1}{|l|}{ ASII } & \multicolumn{1}{|l|}{ SRIL } & \multicolumn{1}{l|}{ IHSG } & \multicolumn{1}{l|}{ RF } \\
\hline Jan-18 & 8500 & 382 & 6650 & $4.25 \%$ \\
\hline Feb-18 & 8075 & 336 & 6600 & $4.25 \%$ \\
\hline Mar-18 & 7300 & 334 & 6690 & $4.25 \%$ \\
\hline Apr-18 & 7150 & 342 & 5650 & $4.50 \%$ \\
\hline Mei-18 & 6900 & 344 & 5650 & $4.75 \%$ \\
\hline Jun-18 & 6600 & 344 & 5620 & $5.25 \%$ \\
\hline Jul-18 & 7150 & 342 & 5800 & $5.25 \%$ \\
\hline Agu-18 & 7250 & 344 & 5900 & $5.50 \%$ \\
\hline Sep-18 & 7350 & 344 & 5650 & $5.75 \%$ \\
\hline Okt-18 & 7900 & 362 & 5650 & $5.75 \%$ \\
\hline Nov-18 & 8550 & 360 & 6150 & $6.00 \%$ \\
\hline Des-18 & 8225 & 358 & 6600 & $6.00 \%$ \\
\hline Jan-19 & 8450 & 340 & 6650 & $6.00 \%$ \\
\hline
\end{tabular}


TABLE 4.8 / Data Closing Price Sektor Infrastruktur

\begin{tabular}{|c|c|c|c|c|c|c|l|}
\hline Bulan & \multicolumn{7}{|c|}{ INFRASTRUKTUR } \\
\hline & \multicolumn{7}{|c|}{ Close Price } \\
\hline & EXCL & INDY & JSMR & PGAS & TLKM & IHSG & RF \\
\hline Jan18 & 2950 & 4470 & 5700 & 2810 & 2990 & 6650 & $4.25 \%$ \\
\hline Feb18 & 2950 & 4220 & 5250 & 2870 & 4000 & 6600 & $4.25 \%$ \\
\hline Mar18 & 2520 & 2520 & 4580 & 2200 & 2600 & 6690 & $4.25 \%$ \\
\hline Apr18 & 2120 & 2410 & 4270 & 1965 & 2520 & 5650 & $4.50 \%$ \\
\hline Mei18 & 2100 & 2740 & 4450 & 2070 & 2520 & 5650 & $4.75 \%$ \\
\hline Jun18 & 2320 & 2420 & 4780 & 1995 & 2750 & 5620 & $5.25 \%$ \\
\hline Jul18 & 2750 & 2800 & 4850 & 1700 & 2570 & 5800 & $5.25 \%$ \\
\hline Agu18 & 2150 & 2270 & 4520 & 2140 & 2480 & 5900 & $5.50 \%$ \\
\hline Sep18 & 2750 & 2800 & 4470 & 2250 & 2640 & 5650 & $5.75 \%$ \\
\hline Okt18 & 2540 & 2260 & 4150 & 2220 & 2550 & 5650 & $5.75 \%$ \\
\hline Nov18 & 1950 & 1800 & 4120 & 1955 & 2680 & 6150 & $6.00 \%$ \\
\hline Des18 & 1875 & 1585 & 4250 & 2120 & 2750 & 6600 & $6.00 \%$ \\
\hline Jan19 & 2170 & 2720 & 4920 & 2570 & 2900 & 6650 & $6.00 \%$ \\
\hline
\end{tabular}


TABLE 4.9 / Data Closing Price Sektor Consumer

\begin{tabular}{|c|c|c|c|c|c|c|c|l|}
\hline Bulan & \multicolumn{7}{|c|}{ Close Price } & \\
\hline & \multicolumn{7}{|c|}{ CONSUMER } \\
\hline & GGRM & HMSP & ICBP & INDF & KLBF & UNVR & IHSG & RF \\
\hline Jan18 & 81000 & 4900 & 8725 & 7750 & 1885 & 54400 & 6650 & $4.25 \%$ \\
\hline Feb18 & 79750 & 4820 & 8975 & 7575 & 1800 & 52900 & 6600 & $4.25 \%$ \\
\hline Mar18 & 72475 & 2960 & 8275 & 7200 & 1500 & 49525 & 6690 & $4.25 \%$ \\
\hline Apr18 & 62225 & 2540 & 8675 & 6975 & 1505 & 48250 & 5650 & $4.50 \%$ \\
\hline Mei18 & 68500 & 2750 & 8700 & 7075 & 1270 & 45800 & 5650 & $4.75 \%$ \\
\hline Jun18 & 87250 & 2580 & 8725 & 4650 & 1220 & 48100 & 5620 & $5.25 \%$ \\
\hline Jul18 & 75150 & 2840 & 8675 & 4250 & 1295 & 42250 & 5800 & $5.25 \%$ \\
\hline Agu18 & 72000 & 2820 & 8525 & 4275 & 1345 & 42350 & 5900 & $5.50 \%$ \\
\hline Sep18 & 74050 & 2850 & 8925 & 5900 & 1380 & 47025 & 5650 & $5.75 \%$ \\
\hline Okt18 & 72200 & 2720 & 8550 & 5975 & 1370 & 42225 & 5650 & $5.75 \%$ \\
\hline Nov18 & 82000 & 2680 & 10450 & 6800 & 1325 & 42250 & 6150 & $6.00 \%$ \\
\hline Des18 & 82625 & 2710 & 10150 & 7450 & 1320 & 45400 & 6600 & $6.00 \%$ \\
\hline Jan19 & 82650 & 2820 & 10775 & 7750 & 1600 & 50000 & 6650 & $6.00 \%$ \\
\hline
\end{tabular}


TABLE 5.0 / Actual Return Perusahaan Property

\begin{tabular}{|c|c|c|c|c|c|c|c|}
\hline \multirow{3}{*}{ Bulan } & \multicolumn{5}{|c|}{ Actual Return } & \multirow{3}{*}{ IHSG } & \multirow{3}{*}{ RF } \\
\hline & \multicolumn{5}{|c|}{ PROPERTY } & & \\
\hline & ADHI & BSDE & PT.PP & WIKA & WSKT & & \\
\hline $\operatorname{Jan} 18$ & 0,1121 & 0,0632 & $\begin{array}{c}- \\
0,0096\end{array}$ & $\begin{array}{c}- \\
0,0745\end{array}$ & 0,0283 & $-0,0075$ & 0,0035 \\
\hline Feb18 & $-0,1653$ & $\begin{array}{c}- \\
0,0801\end{array}$ & $\begin{array}{c}- \\
0,1581\end{array}$ & $\begin{array}{c}- \\
0,1273\end{array}$ & $-0,1$ & 0,0136 & 0,0035 \\
\hline Mar18 & $-0,0918$ & $\begin{array}{c}- \\
0,0506 \\
\end{array}$ & $\begin{array}{c}- \\
0,0690 \\
\end{array}$ & $\begin{array}{c}- \\
0,0565 \\
\end{array}$ & $\begin{array}{c}- \\
0,1053 \\
\end{array}$ & $-0,1555$ & 0,0035 \\
\hline Apr18 & 0,0346 & 0,0089 & 0,0617 & 0,0536 & 0,0498 & 0 & 0,0038 \\
\hline Mei18 & $-0,0797$ & $\begin{array}{c}- \\
0,0821 \\
\end{array}$ & $\begin{array}{c}- \\
0,2267 \\
\end{array}$ & $\begin{array}{c}- \\
0,2066 \\
\end{array}$ & $\begin{array}{c}- \\
0,1703 \\
\end{array}$ & $-0,0053$ & 0,0040 \\
\hline Jun18 & $-0,1034$ & $\begin{array}{c}- \\
0,1374 \\
\end{array}$ & 0,0426 & 0,1698 & 0,1013 & 0,0320 & 0,0044 \\
\hline Jul18 & $-0,0717$ & $\begin{array}{c}- \\
0,1111 \\
\end{array}$ & $\begin{array}{c}- \\
0,0865 \\
\end{array}$ & 0 & $\begin{array}{c}- \\
0,1061 \\
\end{array}$ & 0,0172 & 0,0044 \\
\hline Agu18 & $-0,0671$ & $\begin{array}{c}- \\
0,0375\end{array}$ & $\begin{array}{c}- \\
0,1974\end{array}$ & $\begin{array}{c}- \\
0,1194\end{array}$ & $\begin{array}{c}- \\
0,1029\end{array}$ & $-0,0424$ & 0,0046 \\
\hline Sep18 & $-0,1906$ & $\begin{array}{c}- \\
0,0476 \\
\end{array}$ & $\begin{array}{c}- \\
0,1279 \\
\end{array}$ & $\begin{array}{c}- \\
0,1941 \\
\end{array}$ & $\begin{array}{c}- \\
0,1529 \\
\end{array}$ & 0 & 0,0048 \\
\hline Okt18 & 0,3911 & 0,2273 & 0,3947 & 0,3682 & 0,0833 & 0,0885 & 0,0048 \\
\hline Nov18 & 0,0128 & $\begin{array}{c}- \\
0,0704 \\
\end{array}$ & $\begin{array}{c}- \\
0,0270 \\
\end{array}$ & 0,0997 & 0,0769 & 0,0732 & 0,0050 \\
\hline Des18 & 0,0284 & 0,0598 & 0,2964 & 0,1450 & 0,1756 & 0,0076 & 0,0050 \\
\hline
\end{tabular}


TABLE 5.1 / Actual Return Perusahaan Mining

\begin{tabular}{|c|c|c|c|c|c|c|}
\hline \multirow{3}{*}{ Bulan } & \multicolumn{4}{|c|}{ Actual Return } & \multirow{3}{*}{ IHSG } & \multirow{3}{*}{ RF } \\
\hline & \multicolumn{4}{|c|}{ MINING } & & \\
\hline & ADRO & ANTM & INCO & PTBA & & \\
\hline Jan18 & $-0,0408$ & 0,0437 & $\begin{array}{c}- \\
0,0960 \\
\end{array}$ & $\begin{array}{c}- \\
0,0676 \\
\end{array}$ & $\begin{array}{c}- \\
0,0075 \\
\end{array}$ & 0,0035 \\
\hline Feb18 & $-0,0936$ & $-0,1885$ & $\begin{array}{c}- \\
0,1770 \\
\end{array}$ & $\begin{array}{c}- \\
0,0726 \\
\end{array}$ & 0,0136 & 0,0035 \\
\hline Mar18 & $-0,1385$ & 0,0903 & 0,1326 & 0,1020 & $\begin{array}{c}- \\
0,1555 \\
\end{array}$ & 0,0035 \\
\hline Apr18 & 0,0272 & 0,0237 & 0,2215 & 0,1728 & 0 & 0,0038 \\
\hline Mei18 & $-0,0504$ & 0,0289 & 0,0466 & 0,0447 & $\begin{array}{c}- \\
0,0053 \\
\end{array}$ & 0,0040 \\
\hline Jun18 & 0,0642 & 0,0281 & 0,0817 & 0,1285 & 0,0320 & 0,0044 \\
\hline Jul18 & $-0,0210$ & 4,7049 & $\begin{array}{c}- \\
0,1304 \\
\end{array}$ & $\begin{array}{c}- \\
0,0960 \\
\end{array}$ & 0,0172 & 0,0044 \\
\hline Agu18 & $-0,0161$ & $-0,0287$ & $\begin{array}{c}- \\
0,0237\end{array}$ & 0,0667 & $\begin{array}{c}- \\
0,0424\end{array}$ & 0,0046 \\
\hline Sep18 & $-0,1008$ & $-0,1953$ & $\begin{array}{c}- \\
0,2129\end{array}$ & $\begin{array}{c}- \\
0,0162\end{array}$ & 0 & 0,0048 \\
\hline Okt18 & $-0,2212$ & $-0,0956$ & 0,0377 & $\begin{array}{c}- \\
0,0541 \\
\end{array}$ & 0,0885 & 0,0048 \\
\hline Nov18 & $-0,0545$ & 0,2439 & 0,0759 & 0,0697 & 0,0732 & 0,0050 \\
\hline Des18 & 0,1440 & $-0,5795$ & 0,1810 & 0,0023 & 0,0076 & 0,0050 \\
\hline
\end{tabular}


TABLE 5.2 / Actual Return Trade

\begin{tabular}{|c|c|c|c|c|c|c|c|}
\hline \multirow{3}{*}{ Bulan } & \multicolumn{5}{|c|}{ Actual Return } & \multirow{3}{*}{ IHSG } & \multirow{3}{*}{ RF } \\
\hline & \multicolumn{5}{|c|}{ TRADE } & & \\
\hline & AKRA & LPPF & MNCN & SCMA & UNTR & & \\
\hline Jan18 & $\begin{array}{c}- \\
0,0201\end{array}$ & $\begin{array}{c}- \\
0,0427\end{array}$ & 0,0066 & $\begin{array}{c}- \\
0,0493\end{array}$ & $\begin{array}{c}- \\
0,0848\end{array}$ & $\begin{array}{c}- \\
0,0075\end{array}$ & 0,0035 \\
\hline Feb18 & $\begin{array}{c}- \\
0,0697\end{array}$ & 0,0282 & $\begin{array}{c}- \\
0,0782\end{array}$ & $\begin{array}{c}- \\
0,0667\end{array}$ & $\begin{array}{c}- \\
0,1011\end{array}$ & 0,0136 & 0,0035 \\
\hline Mar18 & $\begin{array}{c}- \\
0,1366 \\
\end{array}$ & $\begin{array}{c}- \\
0,0548 \\
\end{array}$ & $\begin{array}{c}- \\
0,0636 \\
\end{array}$ & $\begin{array}{c}- \\
0,0635 \\
\end{array}$ & 0,0656 & $\begin{array}{c}- \\
0,1555 \\
\end{array}$ & 0,0035 \\
\hline Apr18 & 0,0041 & $\begin{array}{c}- \\
0,1159\end{array}$ & $\begin{array}{c}- \\
0,0943\end{array}$ & $\begin{array}{c}- \\
0,0508\end{array}$ & 0,0279 & 0 & 0,0038 \\
\hline Mei18 & $\begin{array}{c}- \\
0,1260\end{array}$ & $\begin{array}{c}- \\
0,0383\end{array}$ & $\begin{array}{c}- \\
0,2333\end{array}$ & $\begin{array}{c}- \\
0,0536\end{array}$ & $\begin{array}{c}- \\
0,0984\end{array}$ & $\begin{array}{c}- \\
0,0053\end{array}$ & 0,0040 \\
\hline Jun18 & $\begin{array}{c}- \\
0,0209 \\
\end{array}$ & $\begin{array}{c}- \\
0,0881 \\
\end{array}$ & 0,0707 & $\begin{array}{c}- \\
0,0283 \\
\end{array}$ & 0,1155 & 0,0320 & 0,0044 \\
\hline Jul18 & $\begin{array}{c}- \\
0,1425 \\
\end{array}$ & $\begin{array}{c}- \\
0,0654 \\
\end{array}$ & $\begin{array}{c}- \\
0,0812 \\
\end{array}$ & 0 & $\begin{array}{c}- \\
0,0241 \\
\end{array}$ & 0,0172 & 0,0044 \\
\hline Agu18 & 0,0166 & $\begin{array}{c}- \\
0,0767\end{array}$ & $\begin{array}{c}- \\
0,1105\end{array}$ & 0 & $\begin{array}{c}- \\
0,0407\end{array}$ & $\begin{array}{c}- \\
0,0424\end{array}$ & 0,0046 \\
\hline Sep18 & $\begin{array}{c}- \\
0,0572\end{array}$ & $\begin{array}{c}- \\
0,2996\end{array}$ & $\begin{array}{c}- \\
0,0311\end{array}$ & $\begin{array}{c}- \\
0,0718\end{array}$ & 0,0152 & 0 & 0,0048 \\
\hline Okt18 & 0,1272 & $\begin{array}{c}- \\
0,0206\end{array}$ & 0,0128 & 0,2343 & $\begin{array}{c}- \\
0,1791\end{array}$ & 0,0885 & 0,0048 \\
\hline Nov18 & 0,1000 & 0,1789 & $\begin{array}{c}- \\
0,1266\end{array}$ & 0,0508 & $\begin{array}{c}- \\
0,0055\end{array}$ & 0,0732 & 0,0050 \\
\hline Des18 & 0,2238 & 0,2500 & 0,2246 & 0,0484 & $\begin{array}{c}- \\
0,0594\end{array}$ & 0,0076 & 0,0050 \\
\hline
\end{tabular}


TABLE 5.3 / Actual Return Finance

\begin{tabular}{|c|c|c|c|c|c|c|c|}
\hline \multirow{3}{*}{ Bulan } & \multicolumn{5}{|c|}{ Actual Return } & \multirow{3}{*}{ IHSG } & \multirow{3}{*}{ RF } \\
\hline & \multicolumn{5}{|c|}{ FINANCE } & & \\
\hline & BBCA & BBNI & BBRI & BBTN & BMRI & & \\
\hline Jan18 & 0,0198 & 0,0346 & 0,0216 & 0,0219 & 0,0184 & $\begin{array}{c}- \\
0,0075\end{array}$ & 0,0035 \\
\hline Feb18 & 0,0054 & $\begin{array}{c}- \\
0,1080 \\
\end{array}$ & $\begin{array}{c}- \\
0,0476 \\
\end{array}$ & 0,0160 & $\begin{array}{c}- \\
0,0753 \\
\end{array}$ & 0,0136 & 0,0035 \\
\hline Mar18 & $\begin{array}{c}- \\
0,0515\end{array}$ & $\begin{array}{c}- \\
0,0720\end{array}$ & $\begin{array}{c}- \\
0,1056 \\
\end{array}$ & $\begin{array}{c}- \\
0,1816 \\
\end{array}$ & $\begin{array}{c}- \\
0,0717\end{array}$ & $\begin{array}{c}- \\
0,1555\end{array}$ & 0,0035 \\
\hline Apr18 & 0,0271 & 0,0528 & $\begin{array}{c}- \\
0,0435 \\
\end{array}$ & $\begin{array}{c}- \\
0,0193 \\
\end{array}$ & $\begin{array}{c}- \\
0,0105 \\
\end{array}$ & 0 & 0,0038 \\
\hline Mei18 & $\begin{array}{c}- \\
0,0540\end{array}$ & $\begin{array}{c}- \\
0,1681 \\
\end{array}$ & $\begin{array}{c}- \\
0,0779\end{array}$ & $\begin{array}{c}- \\
0,1967 \\
\end{array}$ & $\begin{array}{c}- \\
0,0284 \\
\end{array}$ & $\begin{array}{c}- \\
0,0053\end{array}$ & 0,0040 \\
\hline Jun18 & 0,0838 & 0,0496 & 0,0810 & $\begin{array}{c}- \\
0,0367\end{array}$ & $\begin{array}{c}- \\
0,0292 \\
\end{array}$ & 0,0320 & 0,0044 \\
\hline Jul18 & 0,0655 & 0,0541 & 0,0358 & 0,1653 & 0,0376 & 0,0172 & 0,0044 \\
\hline Agu18 & $\begin{array}{c}- \\
0,0262\end{array}$ & $\begin{array}{c}- \\
0,0513\end{array}$ & $\begin{array}{c}- \\
0,0094\end{array}$ & $\begin{array}{c}- \\
0,0436\end{array}$ & $\begin{array}{c}- \\
0,0254\end{array}$ & $\begin{array}{c}- \\
0,0424\end{array}$ & 0,0046 \\
\hline Sep18 & $\begin{array}{c}- \\
0,0207\end{array}$ & $\begin{array}{c}- \\
0,0101\end{array}$ & 0 & $\begin{array}{c}- \\
0,1939\end{array}$ & 0,0186 & 0 & 0,0048 \\
\hline Okt18 & 0,1015 & 0,1604 & 0,1492 & 0,2594 & 0,0803 & 0,0885 & 0,0048 \\
\hline Nov18 & $\begin{array}{c}- \\
0,0019\end{array}$ & 0,0353 & 0,0110 & $\begin{array}{c}- \\
0,0487\end{array}$ & $\begin{array}{c}- \\
0,0034 \\
\end{array}$ & 0,0732 & 0,0050 \\
\hline Des18 & 0,0837 & 0,0313 & 0,0519 & 0,0787 & 0,0102 & 0,0076 & 0,0050 \\
\hline
\end{tabular}


TABLE 5.4 / Actual Return MISC-IND

\begin{tabular}{|c|c|c|c|c|}
\hline \multirow{2}{*}{ Bulan } & \multicolumn{2}{|c|}{ Actual Return } & \multirow{2}{*}{} & \multirow{2}{*}{ IHSG } \\
\cline { 2 - 3 } & \multicolumn{2}{|c|}{ MISC - IND } & \\
\cline { 2 - 3 } & ASII & SRIL & & \\
\hline Jan18 & $-0,0500$ & $-0,1204$ & $-0,0075$ & 0,0035 \\
\hline Feb18 & $-0,0960$ & $-0,0060$ & 0,0136 & 0,0035 \\
\hline Mar18 & $-0,0205$ & 0,0240 & $-0,1555$ & 0,0035 \\
\hline Apr18 & $-0,0350$ & 0,0058 & 0 & 0,0038 \\
\hline Mei18 & $-0,0435$ & 0 & $-0,0053$ & 0,0040 \\
\hline Jun18 & 0,0833 & $-0,0058$ & 0,0320 & 0,0044 \\
\hline Jul18 & 0,0140 & 0,0058 & 0,0172 & 0,0044 \\
\hline Agu18 & 0,0138 & 0 & $-0,0424$ & 0,0046 \\
\hline Sep18 & 0,0748 & 0,0523 & 0 & 0,0048 \\
\hline Okt18 & 0,0823 & $-0,0055$ & 0,0885 & 0,0048 \\
\hline Nov18 & $-0,0380$ & $-0,0056$ & 0,0732 & 0,0050 \\
\hline Des18 & 0,0274 & $-0,0503$ & 0,0076 & 0,0050 \\
\hline
\end{tabular}


TABLE 5.5 / Actual Return Basic-Ind Tahun 2018

\begin{tabular}{|c|c|c|c|c|c|c|c|c|}
\hline \multirow{3}{*}{ Bulan } & \multicolumn{6}{|c|}{ Actual Return } & \multirow{3}{*}{ IHSG } & \multirow{3}{*}{ RF } \\
\hline & \multicolumn{6}{|c|}{ BASIC-IND } & & \\
\hline & BRPT & INTP & SMGR & WSBP & INKP & TPIA & & \\
\hline Jan & $\begin{array}{c}- \\
0,0076 \\
\end{array}$ & $\begin{array}{c}- \\
0,0336 \\
\end{array}$ & $\begin{array}{c}- \\
0,0022 \\
\end{array}$ & 0,0127 & 0,1590 & $\begin{array}{c}- \\
0,0829 \\
\end{array}$ & $\begin{array}{c}- \\
0,0075 \\
\end{array}$ & 0,0035 \\
\hline Feb & $\begin{array}{c}- \\
0,1183\end{array}$ & $\begin{array}{c}- \\
0,1829\end{array}$ & $\begin{array}{c}- \\
0,0697\end{array}$ & $\begin{array}{c}- \\
0,1375\end{array}$ & 0,0233 & 0,0181 & 0,0136 & 0,0035 \\
\hline Mar & 0,0693 & $\begin{array}{c}- \\
0,0340 \\
\end{array}$ & $\begin{array}{c}- \\
0,0676\end{array}$ & $\begin{array}{c}- \\
0,0242\end{array}$ & 0,1091 & $\begin{array}{c}- \\
0,0414\end{array}$ & $\begin{array}{c}- \\
0,1555\end{array}$ & 0,0035 \\
\hline Apr & $\begin{array}{c}- \\
0,1417 \\
\end{array}$ & 0,0968 & $\begin{array}{c}- \\
0,1295 \\
\end{array}$ & $\begin{array}{c}- \\
0,0050 \\
\end{array}$ & 0,2049 & 0,4444 & 0 & 0,0038 \\
\hline Mei & $\begin{array}{c}- \\
0,0660\end{array}$ & $\begin{array}{c}- \\
0,0856\end{array}$ & $\begin{array}{c}- \\
0,1518\end{array}$ & $\begin{array}{c}- \\
0,0896\end{array}$ & 0,2789 & 0,0085 & $\begin{array}{c}- \\
0,0053\end{array}$ & 0,0040 \\
\hline Jun & $\begin{array}{c}- \\
0,0833 \\
\end{array}$ & 0,0526 & 0,0667 & 0,1694 & 0,0213 & $\begin{array}{c}- \\
0,1379\end{array}$ & 0,0320 & 0,0044 \\
\hline Jul & 0,0551 & 0,0917 & 0,2434 & $\begin{array}{c}- \\
0,0888\end{array}$ & 0,0052 & 0,1000 & 0,0172 & 0,0044 \\
\hline Agu & 0,0729 & $\begin{array}{c}- \\
0,1437 \\
\end{array}$ & 0,0503 & $\begin{array}{c}- \\
0,0821 \\
\end{array}$ & $\begin{array}{c}- \\
0,0969 \\
\end{array}$ & $\begin{array}{c}- \\
0,1055 \\
\end{array}$ & $\begin{array}{c}- \\
0,0424 \\
\end{array}$ & 0,0046 \\
\hline Sep & 0,0190 & $\begin{array}{c}- \\
0,2000 \\
\end{array}$ & $\begin{array}{c}- \\
0,0932 \\
\end{array}$ & $\begin{array}{c}- \\
0,1117 \\
\end{array}$ & $\begin{array}{c}- \\
0,2623 \\
\end{array}$ & $\begin{array}{c}- \\
0,0833 \\
\end{array}$ & 0 & 0,0048 \\
\hline Okt & 0,1253 & $\begin{array}{c}- \\
0,1964 \\
\end{array}$ & 0,3361 & 0,1006 & $\begin{array}{c}- \\
0,1513 \\
\end{array}$ & 0,1585 & 0,0885 & 0,0048 \\
\hline Nov & 0,1327 & $\begin{array}{c}- \\
0,1194 \\
\end{array}$ & $\begin{array}{c}- \\
0,0437\end{array}$ & 0,0743 & 0,0509 & 0 & 0,0732 & 0,0050 \\
\hline Des & 0,0921 & 0,3438 & 0,1022 & 0,0213 & 0,0573 & 0,0670 & 0,0076 & 0,0050 \\
\hline
\end{tabular}


TABLE 5.6 / Actual Return Perusahaan Infrastruktur

\begin{tabular}{|c|c|c|c|c|c|c|c|}
\hline \multirow{3}{*}{ Bulan } & \multirow{2}{*}{\multicolumn{5}{|c|}{$\begin{array}{c}\text { Actual Return } \\
\text { INFRASTRUKTUR }\end{array}$}} & \multirow{3}{*}{ IHSG } & \multirow{3}{*}{ RF } \\
\hline & & & & & & & \\
\hline & EXCL & INDY & JSMR & PGAS & TLKM & & \\
\hline $\begin{array}{l}\text { Jan- } \\
18\end{array}$ & 0,0000 & $\begin{array}{c}- \\
0,0559\end{array}$ & $\begin{array}{c}- \\
0,0789\end{array}$ & 0,0214 & 0,3378 & $\begin{array}{c}- \\
0,0075\end{array}$ & 0,0035 \\
\hline $\begin{array}{c}\text { Feb- } \\
18\end{array}$ & $\begin{array}{c}- \\
0,1458 \\
\end{array}$ & $\begin{array}{c}- \\
0,4028 \\
\end{array}$ & $\begin{array}{c}- \\
0,1276 \\
\end{array}$ & $\begin{array}{c}- \\
0,2334\end{array}$ & $\begin{array}{c}- \\
0,3500\end{array}$ & 0,0136 & 0,0035 \\
\hline $\begin{array}{c}\text { Mar- } \\
18\end{array}$ & $\begin{array}{c}- \\
0,1587\end{array}$ & $\begin{array}{c}- \\
0,0437\end{array}$ & $\begin{array}{c}- \\
0,0677\end{array}$ & $\begin{array}{c}- \\
0,1068\end{array}$ & $\begin{array}{c}- \\
0,0308\end{array}$ & $\begin{array}{c}- \\
0,1555\end{array}$ & 0,0035 \\
\hline $\begin{array}{c}\text { Apr- } \\
18\end{array}$ & $\begin{array}{c}- \\
0,0094\end{array}$ & 0,1369 & 0,0422 & 0,0534 & 0 & 0 & 0,0038 \\
\hline $\begin{array}{c}\text { Mei- } \\
18\end{array}$ & 0,1048 & $\begin{array}{c}- \\
0,1168\end{array}$ & 0,0742 & $\begin{array}{c}- \\
0,0362\end{array}$ & 0,0913 & $\begin{array}{c}- \\
0,0053\end{array}$ & 0,0040 \\
\hline $\begin{array}{c}\text { Jun- } \\
18 \\
\end{array}$ & 0,1853 & 0,1570 & 0,0146 & $\begin{array}{c}- \\
0,1479\end{array}$ & $\begin{array}{c}- \\
0,0655\end{array}$ & 0,0320 & 0,0044 \\
\hline Jul-18 & $\begin{array}{c}- \\
0,2182\end{array}$ & $\begin{array}{c}- \\
0,1893\end{array}$ & $\begin{array}{c}- \\
0,0680\end{array}$ & 0,2588 & $\begin{array}{c}- \\
0,0350\end{array}$ & 0,0172 & 0,0044 \\
\hline $\begin{array}{c}\text { Agu- } \\
18 \\
\end{array}$ & 0,2791 & 0,2335 & $\begin{array}{c}- \\
0,0111 \\
\end{array}$ & 0,0514 & 0,0645 & $\begin{array}{c}- \\
0,0424 \\
\end{array}$ & 0,0046 \\
\hline $\begin{array}{c}\text { Sep- } \\
18 \\
\end{array}$ & $\begin{array}{c}- \\
0,0764 \\
\end{array}$ & $\begin{array}{c}- \\
0,1929 \\
\end{array}$ & $\begin{array}{c}- \\
0,0716 \\
\end{array}$ & $\begin{array}{c}- \\
0,0133 \\
\end{array}$ & $\begin{array}{c}- \\
0,0341 \\
\end{array}$ & 0 & 0,0048 \\
\hline $\begin{array}{c}\text { Okt- } \\
18\end{array}$ & $\begin{array}{c}- \\
0,2323\end{array}$ & $\begin{array}{c}- \\
0,2035\end{array}$ & $\begin{array}{c}- \\
0,0072\end{array}$ & $\begin{array}{c}- \\
0,1194\end{array}$ & 0,0510 & 0,0885 & 0,0048 \\
\hline $\begin{array}{c}\text { Nov- } \\
18\end{array}$ & $\begin{array}{c}- \\
0,0385\end{array}$ & $\begin{array}{c}- \\
0,1194\end{array}$ & 0,0316 & 0,0844 & 0,0261 & 0,0732 & 0,0050 \\
\hline $\begin{array}{c}\text { Des- } \\
18\end{array}$ & 0,1573 & 0,7161 & 0,1576 & 0,2123 & 0,0545 & 0,0076 & 0,0050 \\
\hline
\end{tabular}


TABLE 5.7 / Actual Return Perusahaan Consumer

\begin{tabular}{|c|c|c|c|c|c|c|c|c|}
\hline \multirow{3}{*}{ Bulan } & \multicolumn{6}{|c|}{ Actual return } & \multirow{3}{*}{ IHSG } & \multirow{3}{*}{ RF } \\
\hline & \multicolumn{6}{|c|}{ CONSUMER } & & \\
\hline & GGRM & HMSP & ICBP & INDF & KLBF & UNVR & & \\
\hline $\begin{array}{c}\text { Jan- } \\
18 \\
\end{array}$ & $\begin{array}{c}- \\
0,0154 \\
\end{array}$ & $\begin{array}{c}- \\
0,0163 \\
\end{array}$ & 0,0287 & $\begin{array}{c}- \\
0,0226 \\
\end{array}$ & $\begin{array}{c}- \\
0,0451\end{array}$ & $\begin{array}{c}- \\
0,0276\end{array}$ & $\begin{array}{c}- \\
0,0075\end{array}$ & 0,0035 \\
\hline $\begin{array}{c}\text { Feb- } \\
18 \\
\end{array}$ & $\begin{array}{c}- \\
0,0912\end{array}$ & $\begin{array}{c}- \\
0,3859 \\
\end{array}$ & $\begin{array}{c}- \\
0,0780\end{array}$ & $\begin{array}{c}- \\
0,0495\end{array}$ & $\begin{array}{c}- \\
0,1667\end{array}$ & $\begin{array}{c}- \\
0,0638\end{array}$ & 0,0136 & 0,0425 \\
\hline $\begin{array}{c}\text { Mar- } \\
18\end{array}$ & $\begin{array}{c}- \\
0,1414\end{array}$ & $\begin{array}{c}- \\
0,1419\end{array}$ & 0,0483 & 0,0313 & 0,0033 & $\begin{array}{c}- \\
0,0257\end{array}$ & $\begin{array}{c}- \\
0,1555\end{array}$ & 0,0425 \\
\hline $\begin{array}{c}\text { Apr- } \\
18\end{array}$ & 0,1008 & 0,0827 & 0,0029 & 0,0143 & $\begin{array}{c}- \\
0,1561\end{array}$ & $\begin{array}{c}- \\
0,0508 \\
\end{array}$ & 0 & 0,0450 \\
\hline $\begin{array}{c}\text { Mei- } \\
18 \\
\end{array}$ & 0,2737 & $\begin{array}{c}- \\
0,0618\end{array}$ & 0,0029 & $\begin{array}{c}- \\
0,3428\end{array}$ & $\begin{array}{c}- \\
0,0394\end{array}$ & 0,0502 & $\begin{array}{c}- \\
0,0053\end{array}$ & 0,0475 \\
\hline $\begin{array}{c}\text { Jun- } \\
18 \\
\end{array}$ & $\begin{array}{c}- \\
0,1387 \\
\end{array}$ & 0,1008 & $\begin{array}{c}- \\
0,0057 \\
\end{array}$ & 0,0860 & 0,0615 & $\begin{array}{c}- \\
0,1216 \\
\end{array}$ & 0,0320 & 0,0525 \\
\hline $\begin{array}{c}\text { Jul- } \\
18\end{array}$ & $\begin{array}{c}- \\
0,0419\end{array}$ & $\begin{array}{c}- \\
0,0070\end{array}$ & $\begin{array}{c}- \\
0,0173\end{array}$ & 0,0059 & 0,0386 & 0,0024 & 0,0172 & 0,0525 \\
\hline $\begin{array}{c}\text { Agu- } \\
18\end{array}$ & 0,0285 & 0,0106 & 0,0469 & 0,3801 & 0,0260 & 0,1104 & $\begin{array}{c}- \\
0,0424\end{array}$ & 0,0550 \\
\hline $\begin{array}{c}\text { Sep- } \\
18\end{array}$ & $\begin{array}{c}- \\
0,0250\end{array}$ & $\begin{array}{c}- \\
0,0456\end{array}$ & $\begin{array}{c}- \\
0,0420\end{array}$ & 0,0127 & $\begin{array}{c}- \\
0,0072\end{array}$ & $\begin{array}{c}- \\
0,1021\end{array}$ & 0 & 0,0575 \\
\hline $\begin{array}{c}\text { Okt- } \\
18 \\
\end{array}$ & 0,1357 & $\begin{array}{c}- \\
0,0147 \\
\end{array}$ & 0,2222 & 0,1381 & $\begin{array}{c}- \\
0,0328 \\
\end{array}$ & 0,0006 & 0,0885 & 0,0575 \\
\hline $\begin{array}{c}\text { Nov- } \\
18\end{array}$ & 0,0076 & 0,0112 & $\begin{array}{c}- \\
0,0287\end{array}$ & 0,0956 & $\begin{array}{c}- \\
0,0038 \\
\end{array}$ & 0,0746 & 0,0732 & 0,0600 \\
\hline $\begin{array}{c}\text { Des- } \\
18\end{array}$ & 0,0003 & 0,0406 & 0,0616 & 0,0403 & 0,2121 & 0,1013 & 0,0076 & 0,0600 \\
\hline
\end{tabular}


TABLE 5.8 / Perusahaan Property

\begin{tabular}{|c|c|c|c|c|c|}
\hline & ADHI & BSDE & PT.PP & WIKA & WSKT \\
\hline$E(R)$ & $-0,0159$ & $-0,0215$ & $-0,0089$ & 0,0048 & $-0,0228$ \\
\hline$\sigma^{2}$ & 0,0219539 & 0,0091103 & 0,0327595 & 0,0264597 & 0,0132645 \\
\hline$\alpha$ & $-0,0179045$ & $-0,0222574$ & $-0,011234$ & 0,0021839 & $-0,0243561$ \\
\hline$\beta$ & 1,1285 & 0,4387 & 1,3119 & 1,4740 & 0,8732 \\
\hline$\sigma_{e i}^{2}$ & $-0,0179045$ & $-0,0222574$ & $-0,011234$ & 0,0021839 & $-0,0243561$ \\
\hline ER & 0,1086 & 0,0596 & $-0,0131$ & $-0,0781$ & 0,0247 \\
\hline \multicolumn{2}{|c|}{ Rata-rata ER } & 0,0204 & & & \\
\hline ERB & 0,0180 & 0,0464 & 0,0155 & 0,0138 & 0,0233 \\
\hline $\mathrm{Ai}$ & $-0,0202045$ & $-0,0097633$ & $-0,014739$ & 0,0032189 & $-0,0212676$ \\
\hline $\mathrm{Bi}$ & 0,0177763 & 0,0106783 & 0,0081365 & 0,0003454 & 0,0254542 \\
\hline $\mathrm{Ci}$ & $-0,0198516$ & $-0,0096602$ & $-0,01462$ & 0,0032178 & $-0,0207397$ \\
\hline$C^{*}$ & 0,0032178 & & & & \\
\hline
\end{tabular}


TABLE 5.9 / Perusahaan Mining

\begin{tabular}{|c|c|c|c|c|}
\hline & ADRO & ANTM & INCO & PTBA \\
\hline$E(R)$ & $-0,0418$ & 0,3397 & 0,0114 & 0,0234 \\
\hline$\sigma^{2}$ & 0,0082987 & 1,770252 & 0,018111 & 0,006983 \\
\hline$\alpha$ & $-0,041854$ & 0,336856 & 0,011904 & 0,024104 \\
\hline$\beta$ & 0,0400 & 1,5662 & $-0,2741$ & $-0,4201$ \\
\hline$\sigma_{e i}^{2}$ & $-0,041854$ & 0,336856 & 0,011904 & 0,024104 \\
\hline ER & $-0,0444$ & 0,0402 & $-0,0995$ & $-0,0712$ \\
\hline \multicolumn{2}{|c|}{ Rata-rata ER } & $-0,0437$ & & \\
\hline ERB & $-1,0919$ & $-0,0279$ & 0,1596 & 0,1041 \\
\hline$A i$ & $\mid-0,0017$ & 0,5276 & $-0,0033$ & $-0,0101$ \\
\hline $\mathrm{Bi}$ & $-0,0016$ & $-4,0643$ & 0,0009 & 0,0056 \\
\hline $\mathrm{Ci}$ & $-0,0017$ & $-0,1722$ & $-0,0033$ & $-0,0101$ \\
\hline$C^{*}$ & $-0,001679$ & & & \\
\hline
\end{tabular}


TABLE 5.11 / Perusahaan Finance

\begin{tabular}{|c|c|c|c|c|c|}
\hline & BBCA & BBNI & BBRI & BBTN & BMRI \\
\hline$E(R)$ & 0,0194 & 0,0007 & 0,0055 & $-0,0149$ & $-0,0066$ \\
\hline$\sigma^{2}$ & 0,002662626 & 0,007097 & 0,00454 & 0,017845 & 0,00178 \\
\hline$\alpha$ & 0,01840111 & $-0,0007$ & 0,00406 & $-0,01727$ & $-0,00735$ \\
\hline$\beta$ & 0,5446 & 0,7864 & 0,8319 & 1,3043 & 0,4414 \\
\hline$\sigma_{e i}^{2}$ & 0,01840111 & $-0,0007$ & 0,00406 & $-0,01727$ & $-0,00735$ \\
\hline ER & 0,0163 & 0,0310 & 0,0181 & 0,0183 & 0,0149 \\
\hline \multicolumn{2}{|c|}{ Rata-rata ER } & 0,0197 & & & \\
\hline ERB & 0,0362 & 0,0251 & 0,0237 & 0,0151 & 0,0447 \\
\hline $\mathrm{Ai}$ & 0,010022056 & $-0,00055$ & 0,003378 & $-0,02253$ & $-0,00325$ \\
\hline $\mathrm{Bi}$ & 0,009356274 & $1,98 \mathrm{E}-05$ & 0,000696 & 0,019741 & 0,001211 \\
\hline $\mathrm{Ci}$ & 0,009929156 & $-0,00055$ & 0,003375 & $-0,02209$ & $-0,00324$ \\
\hline$C^{*}$ & 0,009929156 & & & & \\
\hline
\end{tabular}


TABLE 5.12 / Perusahaan Misc-Ind

\begin{tabular}{|c|c|c|}
\hline & ASII & SIRIL \\
\hline$E(R)$ & 0,0010 & $-0,0088$ \\
\hline$\sigma^{2}$ & 0,003093828 & 0,0016342 \\
\hline$\alpha$ & 0,000632332 & $-0,008619$ \\
\hline$\beta$ & 0,2331 & $-0,0999$ \\
\hline$\sigma_{e i}^{2}$ & 0,000632332 & $-0,008619$ \\
\hline ER & $-0,0535$ & $-0,1240$ \\
\hline \multicolumn{2}{|c|}{ Rata-rata ER } & $-0,088$ \\
\hline ERB & $-0,3807$ & 0,8884 \\
\hline $\mathrm{Ai}$ & 0,00014742 & 0,000861 \\
\hline $\mathrm{Bi}$ & $-1,05033 \mathrm{E}-06$ & $8,361 \mathrm{E}-05$ \\
\hline $\mathrm{Ci}$ & 0,00014742 & 0,0008609 \\
\hline C* & 0,000860931 & \\
\hline
\end{tabular}


TABLE 5.13 / Perusahaan Basic-Ind

\begin{tabular}{|c|c|c|c|c|c|c|}
\hline & BRPT & INTP & SMGR & WSBP & INKP & TPIA \\
\hline$E(R)$ & 0,0033 & $-0,0495$ & 0,0201 & $-0,0134$ & 0,0324 & 0,0274 \\
\hline$\sigma^{2}$ & 0,008384 & 0,02216 & 0,020387 & 0,00812 & 0,021028 & 0,02285279 \\
\hline$\alpha$ & 0,003145 & $-0,04894$ & 0,018254 & $-0,01445$ & 0,033496 & 0,026359346 \\
\hline$\beta$ & 0,0674 & $-0,3131$ & 1,0177 & 0,5991 & $-0,6022$ & 0,5662 \\
\hline$\sigma_{e i}^{2}$ & 0,003145 & $-0,04894$ & 0,018254 & $-0,01445$ & 0,033496 & 0,026359 \\
\hline ER & $-0,0111$ & $-0,0371$ & $-0,0058$ & 0,0091 & 0,1555 & $-0,0864$ \\
\hline \multicolumn{2}{|c|}{ Rata-rata ER } & 0,0221 & & & & \\
\hline ERB & 0,3281 & $-0,0707$ & 0,0217 & 0,0369 & $-0,0367$ & 0,0391 \\
\hline $\mathrm{Ai}$ & 0,000212 & 0,015322 & 0,018577 & $-0,00866$ & $-0,02017$ & 0,014924255 \\
\hline $\mathrm{Bi}$ & $3,01 \mathrm{E}-05$ & $-0,0339$ & 0,01533 & 0,005652 & $-0,03055$ & 0,017783783 \\
\hline $\mathrm{Ci}$ & 0,000212 & 0,015859 & 0,018297 & $-0,00861$ & $-0,02081$ & 0,014663 \\
\hline $\mathrm{C}^{*}$ & 0,018297 & & & & & \\
\hline
\end{tabular}


TABLE 5.14 / Perusahaan Infrastruktur

\begin{tabular}{|c|c|c|c|c|c|}
\hline & EXCL & INDY & JSMR & PGAS & TLKM \\
\hline$E(R)$ & $-0,0127$ & $-0,0067$ & $-0,0093$ & 0,0021 & 0,0092 \\
\hline$\sigma^{2}$ & 0,02511823 & 0,076653 & 0,005777 & 0,019049 & 0,021831 \\
\hline$\alpha$ & $-0,0123116$ & $-0,00555$ & $-0,00985$ & 0,001691 & 0,009237 \\
\hline$\beta$ & $-0,2312$ & $-0,6618$ & 0,2898 & 0,2006 & $-0,0444$ \\
\hline$\sigma_{e i}^{2}$ & $-0,0123116$ & $-0,00555$ & $-0,00985$ & 0,001691 & 0,009237 \\
\hline ER & $-0,0035$ & $-0,0559$ & $-0,0789$ & 0,2526 & 0,9996 \\
\hline \multicolumn{2}{|c|}{ Rata-rata ER } & 0,2228 & & & \\
\hline ERB & $-0,9635$ & $-0,3366$ & 0,7688 & 1,1102 & $-5,0200$ \\
\hline $\mathrm{Ai}$ & 0,00284643 & 0,003673 & $-0,00286$ & 0,000339 & $-0,00041$ \\
\hline $\mathrm{Bi}$ & $-0,0001573$ & $-9,1 \mathrm{E}-05$ & 0,000126 & $2,58 \mathrm{E}-06$ & $-1,7 \mathrm{E}-05$ \\
\hline $\mathrm{Ci}$ & 0,00284688 & 0,003673 & $-0,00285$ & 0,000339 & $-0,00041$ \\
\hline$C^{*}$ & 0,00367314 & & & & \\
\hline
\end{tabular}


TABLE 5.15 / Perusahaan Consumer

\begin{tabular}{|c|c|c|c|c|c|c|}
\hline & BRPT & INTP & SMGR & WSBP & INKP & TPIA \\
\hline$E(R)$ & 0,0078 & $-0,0356$ & 0,0201 & 0,0129 & $-0,0091$ & $-0,0043$ \\
\hline$\sigma^{2}$ & 0,01282 & 0,014928 & 0,005224 & 0,025128 & 0,008903 & 0,005299532 \\
\hline$\alpha$ & 0,006635 & $-0,03652$ & 0,019866 & 0,012613 & $-0,00904$ & $-0,004385396$ \\
\hline$\beta$ & 0,6241 & 0,5028 & 0,1551 & 0,1632 & $-0,0513$ & 0,0223 \\
\hline$\sigma_{e i}^{2}$ & 0,006635 & $-0,03652$ & 0,019866 & 0,012613 & $-0,00904$ & $-0,004385$ \\
\hline ER & $-0,0579$ & $-0,0588$ & $-0,0138$ & $-0,0651$ & $-0,0876$ & $-0,0701$ \\
\hline \multicolumn{2}{|c|}{ Rata-rata ER } & $-0,0567$ & & & & \\
\hline ERB & $-0,0908$ & $-0,1127$ & $-0,3654$ & $-0,3471$ & 1,1047 & $-2,5392$ \\
\hline $\mathrm{Ai}$ & 0,004141 & $-0,01836$ & 0,00308 & 0,002059 & 0,000464 & $-0,000097849$ \\
\hline $\mathrm{Bi}$ & $-0,00048$ & $-0,01183$ & $-0,00108$ & $-0,00046$ & 7,4E-05 & $-0,000007574$ \\
\hline $\mathrm{Ci}$ & 0,004143 & $-0,01858$ & 0,003084 & 0,00206 & 0,000464 & $-0,000098$ \\
\hline$C^{*}$ & 0,004143 & & & & & \\
\hline
\end{tabular}


TABLE 5.16 / Perusahaan Property

\begin{tabular}{|c|c|c|c|c|c|c|c|c|c|c|c|c|}
\hline Emiten & $\alpha$ & $\beta$ & $\sigma_{e i}^{2}$ & ERB & $\mathrm{Ci}$ & $c^{*}$ & Kesimpulan & $\mathrm{Zi}$ & $\mathrm{Wi}$ & $a_{p i}$ & $\beta_{p i}$ & $\sigma_{e p}^{2}$ \\
\hline ADHI & $-0,0179045$ & 1,1285 & $-0,01790$ & 0,0180 & $-0,0198516$ & 0,003218 & Optimal & $-0,9338$ & $-0,2913$ & 0,005215 & $-0,32867$ & 0,005215 \\
\hline BSDE & $-0,0222574$ & 0,4387 & $-0,02226$ & 0,0464 & $-0,0096602$ & 0,003218 & Optimal & $-0,8509$ & $-0,2654$ & 0,005907 & $-0,11642$ & 0,005907 \\
\hline PT.PP & $-0,0112344$ & 1,3119 & $-0,01123$ & 0,0155 & $-0,0146196$ & 0,003218 & Optimal & $-1,4356$ & $-0,4478$ & 0,005031 & $\begin{array}{l}-0,58747 \\
\end{array}$ & 0,005031 \\
\hline WIKA & 0,0021839 & 1,4740 & 0,00218 & 0,0138 & 0,0032178 & 0,003218 & Optimal & 7,1465 & 2,2291 & 0,004868 & 3,285567 & 0,004868 \\
\hline WSKT & $-0,0243561$ & 0,8732 & $-0,02436$ & 0,0233 & $-0,0207397$ & 0,003218 & Optimal & $-0,7202$ & $-0,2246$ & 0,005471 & $-0,19614$ & 0,005471 \\
\hline \multicolumn{5}{|c|}{ Maximum } & 0,0032178 & - & - & 3,2061 & & 0,026492 & 2,056867 & 0,026492 \\
\hline & & & & & & & & & \multicolumn{2}{|c|}{$E(R m)$} & \multicolumn{2}{|c|}{0,0018} \\
\hline & & & & & & & & & \multicolumn{2}{|c|}{ SIM=E(Rp) } & \multicolumn{2}{|c|}{0,03018} \\
\hline & & & & & & & & & \multicolumn{2}{|c|}{$\sigma_{m}^{2}$} & \multicolumn{2}{|c|}{0,00340} \\
\hline & & & & & & & & & \multicolumn{2}{|c|}{$\sigma_{p}^{2}$} & \multicolumn{2}{|c|}{0,040862} \\
\hline
\end{tabular}


TABLE 5.17 / Perusahaan Mining

\begin{tabular}{|c|c|c|c|c|c|c|c|c|c|c|c|c|}
\hline Emiten & $a$ & $\beta$ & $\sigma_{e i}^{2}$ & ERB & $\mathrm{Ci}$ & $c^{*}$ & Kesimpulan & $\mathrm{Zi}$ & Wi & $a_{p i}$ & $\beta_{p i}$ & $\sigma_{e p}^{2}$ \\
\hline ADRO & $-0,041854$ & 0,0400 & $-0,04185$ & $-1,0919$ & $-0,0017$ & $-0,0017$ & - & 1,0432 & $-0,2251$ & 0,009422 & $-0,00901$ & 0,009422 \\
\hline ANTM & 0,3368555 & 1,5662 & 0,33686 & $-0,0279$ & $-0,1722$ & $-0,0017$ & - & $-0,1220$ & 0,0263 & 0,008869 & 0,041236 & 0,008869 \\
\hline INCO & 0,0119044 & $-0,2741$ & 0,01190 & 0,1596 & $-0,0033$ & $-0,0017$ & Optimal & $-3,7120$ & 0,8010 & 0,009535 & $-0,21952$ & 0,009535 \\
\hline PTBA & 0,0241039 & $-0,4201$ & 0,02410 & 0,1041 & $-0,0101$ & $-0,0017$ & Optimal & $-1,8434$ & 0,3978 & 0,009588 & $-0,16711$ & 0,009588 \\
\hline \multicolumn{5}{|c|}{ Maximum } & $-0,0017$ & & & $-4,6342$ & 1,0000 & 0,037414 & $-0,35441$ & 0,037414 \\
\hline & & & & & & & & & \multicolumn{2}{|c|}{$E(R m)$} & 0,0043 & \\
\hline & & & & & & & & & \multicolumn{2}{|c|}{$S I M=E(R p)$} & 0,03590 & \\
\hline & & & & & & & & & \multicolumn{2}{|l|}{$\sigma_{m}^{2}$} & 0,00340 & \\
\hline & & & & & & & & & \multicolumn{2}{|l|}{$\sigma_{p}^{2}$} & 0,037840 & \\
\hline
\end{tabular}


TABLE 5.18 / Perusahaan Trade

\begin{tabular}{|c|c|c|c|c|c|c|c|c|c|c|c|c|}
\hline Emiten & $\alpha$ & $\beta$ & $\sigma_{e i}^{2}$ & ERB & $\mathrm{Ci}$ & $\mathrm{C}^{*}$ & Kesimpulan & $\mathrm{Zi}$ & Wi & $a_{p i}$ & $\beta_{p i}$ & $\sigma_{e p}^{2}$ \\
\hline AKRA & $-0,0101$ & 0,9253 & $-0,01010$ & $-0,04497$ & $-0,0094$ & 0,0155 & - & 5,5393 & 0,3100 & $-0,0031$ & 0,2868 & $-0,0031$ \\
\hline LPPF & $-0,02974$ & 0,5522 & $-0,02974$ & $-0,07536$ & $-0,0166$ & 0,0155 & - & 1,6874 & 0,0944 & $-0,0028$ & 0,0521 & $-0,0028$ \\
\hline MNCN & $-0,04245$ & 0,2461 & $-0,04245$ & \begin{tabular}{|l|}
$-0,16905$ \\
\end{tabular} & $-0,0106$ & 0,0155 & - & 1,0701 & 0,0599 & $-0,0025$ & 0,0147 & $-0,0025$ \\
\hline SCMA & $-0,00568$ & 0,8249 & $-0,00568$ & \begin{tabular}{|l|}
$-0,05044$ \\
\end{tabular} & $-0,0047$ & 0,0155 & - & 9,5728 & $\begin{array}{ll}0,5357 \\
\end{array}$ & $-0,0030$ & 0,4419 & $-0,0030$ \\
\hline UNTR & $-0,02981$ & $-0,5263$ & $-0,02981$ & 1,395882 & 0,0155 & 0,0155 & Optimal & 24,3686 & $\begin{array}{ll}1,3637 \\
\end{array}$ & $-0,0407$ & $-0,7177$ & $-0,0407$ \\
\hline \multicolumn{5}{|c|}{ Maximum } & 0,0155 & & & 17,8696 & 1,0000 & $-0,0115$ & 0,7956 & $-0,0115$ \\
\hline & & & & & & & & & & $E(R m)$ & 0,0018 & \\
\hline & & & & & & & & & & SIM=E(Rp) & $-0,01010$ & \\
\hline & & & & & & & & & & $\sigma_{m}^{2}$ & 0,00340 & \\
\hline & & & & & & & & & & $\sigma_{p}^{2}$ & $-0,0094$ & \\
\hline
\end{tabular}


TABLE 5.19/ Perusahaan Finance

\begin{tabular}{|c|c|c|c|c|c|c|c|c|c|c|c|c|}
\hline Emiten & $\alpha$ & $\beta$ & $\frac{\sigma_{e i}^{2}}{2}$ & ERB & $\mathrm{Ci}$ & $c^{*}$ & \begin{tabular}{|l|} 
Kesimpulan \\
\end{tabular} & $\mathrm{Zi}$ & Wi & $a_{p i}$ & $\beta_{p i}$ & $\sigma_{e p}^{2}$ \\
\hline$B B C A$ & 0,018401 & 0,5446 & 0,01840 & 0,03619 & 0,0099 & 0,0099 & Optimal & 0,7773 & 0,0053 & 0,0001 & 0,0029 & 0,0001 \\
\hline BBNI & $-0,0007$ & 0,7864 & $-0,00070$ & 0,025063 & $-0,0006$ & 0,0099 & Optimal & $-16,8967$ & $-0,1143$ & 0,0001 & $-0,0899$ & 0,0001 \\
\hline BBRI & 0,00406 & 0,8319 & 0,00406 & 0,023693 & 0,0034 & 0,0099 & Optimal & 2,8203 & 0,0191 & 0,0001 & 0,0159 & 0,0001 \\
\hline BBTN & $-0,01727$ & 1,3043 & $-0,01727$ & 0,015112 & $-0,0221$ & 0,0099 & Optimal & $-0,3914$ & $-0,0026$ & 0,0000 & $-0,0035$ & 0,0000 \\
\hline BMRl & $-0,00735$ & 0,414 & $-0,00735$ & $-2,68071$ & $-0,0032$ & 0,0099 & . & 161,5079 & 1,0926 & $-0,0080$ & 0,4822 & $-0,0080$ \\
\hline \multicolumn{5}{|c|}{ Maximum } & 0,0099 & & & 147,8174 & 1,0000 & $-0,0077$ & 0,4076 & $-0,0077$ \\
\hline & & & & & & & & & & $E(R m)$ & 0,0018 & \\
\hline & & & & & & & & & & SIM=E(Rp) & $-0,00700$ & \\
\hline & & & & & & & & & & $\sigma_{m}^{2}$ & 0,00340 & \\
\hline & & & & & & & & & & $\sigma_{p}^{2}$ & $-0,0072$ & \\
\hline
\end{tabular}


TABLE 5.20 / Perusahaan Misc-Ind

\begin{tabular}{|c|c|c|c|c|c|c|c|c|c|c|c|c|}
\hline Emiten & $a$ & $\beta$ & $\sigma_{e i}^{2}$ & ERB & $\mathrm{Ci}$ & $C^{*}$ & Kesimpulan & $2 i$ & Wi & $a_{p i}$ & $\beta_{p i}$ & $\sigma_{e p}^{2}$ \\
\hline ASHI & 0,000632 & 0,2331 & 0,00063 & $-0,3806822$ & 0,0001 & 0,0001 & - & $-140,4096$ & 1,0791 & 0,0007 & 0,2516 & 0,0007 \\
\hline SIRIL & $-0,00862$ & $-0,0999$ & $-0,00862$ & 0,8884046 & 0,0000 & 0,0001 & Optimal & 10,2958 & $-0,0791$ & 0,0007 & 0,0079 & 0,0007 \\
\hline \multicolumn{5}{|c|}{ Maximum } & 0,0001 & & & $-130,1138$ & 1,0000 & 0,0014 & 0,2595 & 0,0014 \\
\hline & & & & & & & & & & $E(R m)$ & 0,0018 & \\
\hline & & & & & & & & & & SIM $=E(R p)$ & 0,00183 & \\
\hline & & & & & & & & & & $\sigma_{m}^{2}$ & 0,00340 & \\
\hline & & & & & & & & & & $\sigma_{p}^{2}$ & 0,0016 & \\
\hline
\end{tabular}


TABLE 2.21 / Perusahaan Basic Ind

\begin{tabular}{|c|c|c|c|c|c|c|c|c|c|c|c|c|}
\hline Emiten & $\alpha$ & $\beta$ & $\sigma_{e i}^{2}$ & ERB & $\mathrm{Ci}$ & $\mathrm{C}^{*}$ & esimpulal & $\mathrm{Zi}$ & Wi & $a_{p i}$ & $\beta_{p i}$ & $\sigma_{e p}^{2}$ \\
\hline BRPT & 0,003145338 & 0,0674 & 0,00315 & 0,328145 & 0,0002 & 0,0183 & Optimal & 6,6408 & 0,0493 & 0,0002 & 0,0033 & 0,0002 \\
\hline INTP & $-0,04893926$ & $-0,3131$ & $-0,04894$ & $-0,07066$ & 0,0159 & 0,0183 & - & $-0,5691$ & $-0,0042$ & 0,0002 & 0,0013 & 0,0002 \\
\hline SMGR & 0,018254221 & 1,0177 & 0,01825 & 1,211825 & 0,0183 & 0,0183 & \begin{tabular}{|l|} 
Optimal \\
\end{tabular} & 66,5408 & 0,4942 & 0,0090 & 0,5029 & 0,0090 \\
\hline WSBP & $-0,01444611$ & 0,5991 & $-0,01445$ & $-1,53127$ & $-0,0086$ & 0,0183 & - & 64,2675 & 0,4773 & $-0,0069$ & 0,2860 & $-0,0069$ \\
\hline INKP & 0,033496384 & $-0,6022$ & 0,03350 & 0,142268 & $-0,0208$ & 0,0183 & Optimal & $-2,2288$ & $-0,0166$ & $-0,0006$ & 0,0100 & $-0,0006$ \\
\hline TPIA & 0,026359346 & 0,5662 & 0,02636 & $-0,25599$ & 0,0147 & 0,0183 & & $-5,8914$ & $-0,0438$ & $-0,0012$ & $-0,0248$ & $-0,0012$ \\
\hline \multicolumn{5}{|c|}{ Maximum } & 0,0183 & & & 134,6512 & 1,0000 & 0,0019 & 0,8035 & 0,0019 \\
\hline & & & & & & & & & & $E(\mathrm{Rm})$ & 0,0018 & \\
\hline & & & & & & & & & & SIM=E(Rp) & 0,00337 & \\
\hline & & & & & & & & & & $\sigma_{m}^{2}$ & 0,00340 & \\
\hline & & & & & & & & & & $\sigma_{p}^{2}$ & 0,0041 & \\
\hline
\end{tabular}


TABLE 5.22 / Perusahaan Infrastruktur

\begin{tabular}{|c|c|c|c|c|c|c|c|c|c|c|c|c|}
\hline Emiten & $\alpha$ & $\beta$ & $\frac{\sigma_{e i}^{2}}{2}$ & ERB & $\mathrm{Ci}$ & $c^{*}$ & Kesimpulan & $2 i$ & $\mathrm{Wi}$ & $a_{p i}$ & $\overline{\beta_{p i}}$ & $\overline{\sigma_{e p}^{2}}$ \\
\hline EXCL & \begin{tabular}{|l|l|} 
& $-0,1231$ \\
\end{tabular} & $-0,2312$ & $-0,01231$ & $-0,96347$ & 0,0028 & 0,0037 & 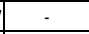 & $-18,1621$ & $-0,3629$ & 0,0045 & 0,0839 & 0,0045 \\
\hline INDY & \begin{tabular}{|l|l|} 
\\
\end{tabular} & $\begin{array}{l}-0,6618 \\
\end{array}$ & $-0,00555$ & \begin{tabular}{|c|}
$-0,33657$ \\
\end{tabular} & $\begin{array}{l}0,0037 \\
\end{array}$ & 0,0037 & 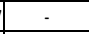 & $-40,5791$ & $-0,8107$ & 0,0045 & 0,5366 & 0,0045 \\
\hline $\begin{array}{l}\text { JSMR } \\
\end{array}$ & \begin{tabular}{|l|l|} 
\\
\end{tabular} & 0,2898 & $-0,00985$ & 0,76878 & $-0,0029$ & 0,0037 & Optimal & $-22,4985$ & $-0,4495$ & 0,0044 & $-0,1302$ & 0,0044 \\
\hline $\begin{array}{l}\text { PGAS } \\
\end{array}$ & \begin{tabular}{|l|l|}
0,001691 \\
\end{tabular} & 0,2006 & \begin{tabular}{|l|l|l|l|l|}
0 \\
\end{tabular} & 1,110208 & 0,0003 & 0,0037 & Optimal & 131,2938 & 2,6230 & 0,0044 & \begin{tabular}{|l|l|}
0,5263 \\
\end{tabular} & 0,0044 \\
\hline TLKM & \begin{tabular}{|l|l|}
0,009237 \\
\end{tabular} & $-0,0444$ & 0,00924 & 24,11567 & $-0,0004$ & 0,0037 & Optimal & $-115,8323$ & $-2,3141$ & $-0,0214$ & 0,1027 & $-0,021$ \\
\hline \multicolumn{5}{|c|}{ Maximum } & 0,0037 & & & 50,0540 & 1,0000 & 0,0178 & 1,0165 & 0,0178 \\
\hline & & & & & & & & & & $E(R m)$ & 0,0018 & \\
\hline & & & & & & & & & & $S I M=E(R p)$ & 0,01965 & \\
\hline & & & & & & & & & & $\sigma_{m}^{2}$ & 0,00340 & \\
\hline & & & & & & & & & & $\sigma_{p}^{2}$ & 0,0213 & \\
\hline
\end{tabular}


TABLE 5.23 / Perusahaan Consumer

\begin{tabular}{|c|c|c|c|c|c|c|c|c|c|c|c|c|}
\hline Emiten & $\alpha$ & $\beta$ & $\sigma_{e i}^{2}$ & ERB & $\mathrm{Ci}$ & $\mathrm{C}^{*}$ & Kesimpulan & $\mathrm{Zi}$ & Wi & $a_{p i}$ & $\beta_{p i}$ & $\sigma_{e p}^{2}$ \\
\hline GGRM & 0,006635 & 0,6241 & 0,00664 & $-0,09078$ & 0,0041 & 0,004 & - & $-8,9286$ & 0,1061 & 0,0007 & 0,0662 & 0,0007 \\
\hline HMSP & $-0,03652$ & 0,5028 & $-0,03652$ & $-0,11269$ & $-0,0186$ & 0,004 & - & 1,6085 & $-0,0191$ & 0,0007 & $-0,0096$ & 0,0007 \\
\hline ICBP & 0,019866 & 0,1551 & 0,01987 & $-2,85188$ & 0,0031 & 0,004 & - & $-22,2914$ & 0,2649 & 0,0053 & 0,0411 & 0,0053 \\
\hline INDF & 0,012613 & 0,1632 & 0,01261 & $-4,49177$ & 0,0021 & 0,004 & - & $-58,1827$ & 0,6914 & 0,0087 & 0,1129 & 0,0087 \\
\hline KLBF & $-0,00904$ & $-0,0513$ & $-0,00904$ & 0,646808 & 0,0005 & 0,004 & Optimal & 3,6462 & $-0,0433$ & 0,0004 & 0,0022 & 0,0004 \\
\hline UNVR & $-0,00439$ & 0,0223 & $-0,00439$ & 0,808519 & $-0,0001$ & 0,004 & & $-4,0926$ & 0,0486 & $-0,0002$ & 0,0011 & $-0,0002$ \\
\hline \multicolumn{5}{|c|}{ Maximum } & 0,0041 & & & $-84,1481$ & 1,0000 & 0,0158 & 0,2128 & 0,0158 \\
\hline & & & & & & & & & & $\mathrm{E}(\mathrm{Rm})$ & 0,0018 & \\
\hline & & & & & & & & & & SIM=E(Rp) & 0,01616 & \\
\hline & & & & & & & & & & $\sigma_{m}^{2}$ & 0,00340 & \\
\hline & & & & & & & & & & $\sigma_{p}^{2}$ & 0,0159 & \\
\hline
\end{tabular}


TABLE 5.24 / Perusahaan Property

\begin{tabular}{|c|c|c|c|c|c|c|c|}
\hline Emiten & $\mathbf{E}(\mathbf{R i})$ & $\boldsymbol{\sigma i}^{\mathbf{2}}$ & $\mathbf{C o v}$ & $\boldsymbol{\rho}$ & $\mathbf{E}(\mathbf{R p})$ & $\boldsymbol{\sigma}_{\boldsymbol{p}}{ }^{2}$ & $\mathbf{W i}$ \\
\hline ADHI & $-0,0684$ & 0,0865 & 0,0849 & 0,9672 & 0,01992 & 0,2909 & $-0,2913$ \\
\hline BSDE & $-0,0930$ & 0,0759 & 0,0865 & 0,9231 & 0,02467 & 0,30171 & $-0,2654$ \\
\hline PTPP & $-0,0602$ & 0,0986 & 0,1014 & 0,9683 & 0,02696 & 0,31715 & $-0,4478$ \\
\hline WIKA & $-0,0462$ & 0,0947 & 0,0879 & 0,9574 & $-0,10306$ & 0,29786 & 2,2291 \\
\hline WSKT & $-0,0585$ & 0,0758 & 0,0802 & 0,9141 & 0,01313 & 0,29063 & $-0,2246$ \\
\hline
\end{tabular}


TABLE 5.25 / Perusahaan Trade

\begin{tabular}{|c|c|c|c|c|c|c|c|}
\hline Emiten & $\mathbf{E}(\mathbf{R i})$ & $\boldsymbol{\sigma} \boldsymbol{i}^{\mathbf{2}}$ & $\mathbf{C o v}$ & $\boldsymbol{\rho}$ & $\mathbf{E}(\mathbf{R p})$ & $\boldsymbol{\sigma}_{\boldsymbol{p}}{ }^{\mathbf{2}}$ & $\mathbf{W i}$ \\
\hline AKRA & $-0,0705$ & 0,0795 & 0,0795 & 0,9302 & $-0,0218$ & 0,2865 & 0,3100 \\
\hline LPPF & $-0,0554$ & 0,0783 & 0,0717 & 0,8701 & $-0,0052$ & 0,2822 & 0,0944 \\
\hline MNCN & $-0,0991$ & 0,0739 & 0,0727 & 0,9121 & $-0,0059$ & 0,2781 & 0,0599 \\
\hline SCMA & $-0,0437$ & 0,0733 & 0,0676 & 0,8716 & $-0,0234$ & 0,2741 & 0,5357 \\
\hline UNTR & $-0,0929$ & 0,0700 & 0,0697 & 0,8624 & $-0,1267$ & 0,2790 & 1,3637 \\
\hline
\end{tabular}


TABLE 5.26 / Perusahaan Mining

\begin{tabular}{|c|c|c|c|c|c|c|c|}
\hline Emiten & $\mathbf{E}(\mathbf{R i})$ & $\boldsymbol{\sigma i}^{\mathbf{2}}$ & $\mathbf{C o v}$ & $\boldsymbol{\rho}$ & $\mathbf{E}(\mathbf{R p})$ & $\boldsymbol{\sigma}_{\boldsymbol{p}}{ }^{2}$ & $\mathbf{W i}$ \\
\hline ADRO & $-0,0801$ & 0,0668 & 0,0939 & 0,2523 & 0,01803 & 0,99003 & $-0,2251$ \\
\hline ANTM & 0,2466 & 1,7648 & 0,0433 & 0,1027 & 0,00648 & 0,99097 & 0,0263 \\
\hline INCO & $-0,0283$ & 0,0860 & 0,0810 & 0,9494 & $-0,02264$ & 0,28881 & 0,8010 \\
\hline PTBA & 0,0401 & 0,0721 & 0,0691 & 0,9188 & 0,01597 & 0,27567 & 0,3978 \\
\hline
\end{tabular}


TABLE 5.27 / Perusahaan Finance

\begin{tabular}{|c|c|c|c|c|c|c|c|}
\hline Emiten & $\mathbf{E}(\mathbf{R i})$ & $\boldsymbol{\sigma i}^{\mathbf{2}}$ & $\mathbf{C o v}$ & $\boldsymbol{\rho}$ & $\mathbf{E}(\mathbf{R p})$ & $\boldsymbol{\sigma}_{\boldsymbol{p}}{ }^{\mathbf{2}}$ & $\mathbf{W i}$ \\
\hline BBCA & $-0,0552$ & 0,0759 & 0,0803 & 0,9821 & $-0,00029$ & 0,28021 & 0,0053 \\
\hline BBNI & $-0,0500$ & 0,0751 & 0,0786 & 0,9860 & 0,00572 & 0,27684 & $-0,1143$ \\
\hline BBRI & $-0,0297$ & 0,0721 & 0,0795 & 0,9484 & $-0,00057$ & 0,28483 & 0,0191 \\
\hline BBTN & $-0,0703$ & 0,0831 & 0,0752 & 0,9270 & 0,00018 & 0,28005 & $-0,0026$ \\
\hline BMRI & $-0,0472$ & 0,0675 & 0,0766 & 0,9882 & $-0,05152$ & 0,27306 & 1,0926 \\
\hline
\end{tabular}


TABLE 5.28 / Perusahaan Misc-Ind

\begin{tabular}{|c|c|c|c|c|c|c|c|}
\hline Emiten & $\mathrm{E}(\mathrm{Ri})$ & $\boldsymbol{\sigma i}^{\mathbf{2}}$ & $\mathbf{C o v}$ & $\boldsymbol{\rho}$ & $\mathrm{E}(\mathrm{Rp})$ & $\boldsymbol{\sigma}_{\boldsymbol{p}}{ }^{\mathbf{2}}$ & $\mathbf{W i}$ \\
\hline ASII & $-0,0675$ & 0,0732 & 0,0758 & 0,9780 & $-0,0728$ & 0,2729 & 1,0791 \\
\hline SRIL & $-0,0757$ & 0,0700 & 0,0758 & 0,9780 & 0,0060 & 0,2729 & $-0,0791$ \\
\hline
\end{tabular}


TABLE 5.29 / Perusahaan Basic Ind

\begin{tabular}{|c|c|c|c|c|c|c|c|}
\hline Emiten & $\mathbf{E}(\mathbf{R i )}$ & $\boldsymbol{\sigma i}^{\mathbf{2}}$ & $\mathbf{C o v}$ & $\boldsymbol{\rho}$ & $\mathbf{E}(\mathbf{R p})$ & $\boldsymbol{\sigma}_{\boldsymbol{p}}{ }^{2}$ & $\mathbf{W i}$ \\
\hline BRPT & $-0,0669$ & 0,0787 & 0,0516 & 0,6924 & $-0,00330$ & 0,28077 & 0,0493 \\
\hline INTP & 0,0933 & 0,0601 & 0,0507 & 0,6317 & $-0,00039$ & 0,28833 & $-0,0042$ \\
\hline SMGR & $-0,0358$ & 0,0915 & 0,0772 & 0,8956 & $-0,01768$ & 0,28902 & 0,4942 \\
\hline WSBP & $-0,0272$ & 0,0691 & 0,0730 & 0,8363 & $-0,01298$ & 0,29132 & 0,4773 \\
\hline INKP & $-0,0387$ & 0,0940 & 0,0864 & 0,8415 & 0,00064 & 0,31401 & $-0,0166$ \\
\hline TPIA & $-0,0464$ & 0,0956 & 0,0749 & 0,7969 & 0,00203 & 0,30122 & $-0,0438$ \\
\hline
\end{tabular}


TABLE 5.30 / Perusahaan Infrastruktur

\begin{tabular}{|c|c|c|c|c|c|c|c|}
\hline Emiten & $\mathbf{E}(\mathbf{R i})$ & $\boldsymbol{\sigma i}^{\mathbf{2}}$ & $\mathbf{C o v}$ & $\boldsymbol{\rho}$ & $\mathbf{E}(\mathbf{R p})$ & $\boldsymbol{\sigma}_{\boldsymbol{p}}{ }^{\mathbf{2}}$ & $\mathbf{W i}$ \\
\hline EXCL & $-0,0591$ & 0,0887 & 0,1033 & 0,8542 & 0,0214 & 0,3452 & $-0,3629$ \\
\hline INDY & $-0,0598$ & 0,1404 & 0,0901 & 0,8167 & 0,0485 & 0,3339 & $-0,8107$ \\
\hline JSMR & $-0,0749$ & 0,0739 & 0,0789 & 0,9086 & 0,0337 & 0,2895 & $-0,4495$ \\
\hline PGAS & $-0,0471$ & 0,0869 & 0,0813 & 0,8673 & $-0,1236$ & 0,3007 & 2,6230 \\
\hline TLKM & $-0,0040$ & 0,0860 & 0,0772 & 0,8153 & 0,0093 & 0,3028 & $-2,3141$ \\
\hline
\end{tabular}


TABLE 5.31 / Perusahaan Consumer

\begin{tabular}{|c|c|c|c|c|c|c|c|}
\hline Emiten & $\mathbf{E}(\mathbf{R i})$ & $\boldsymbol{\sigma i}^{\mathbf{2}}$ & $\mathbf{C o v}$ & $\boldsymbol{\rho}$ & $\mathbf{E}(\mathbf{R p})$ & $\boldsymbol{\sigma}_{\boldsymbol{p}}{ }^{\mathbf{2}}$ & $\mathbf{W i}$ \\
\hline GGRM & $-0,0330$ & 0,0804 & 0,0727 & 0,8631 & $-0,0035$ & 0,2849 & 0,1061 \\
\hline HMSP & $-0,0668$ & 0,0752 & 0,0748 & 0,9010 & 0,0013 & 0,2824 & $-0,0191$ \\
\hline ICBP & $-0,0515$ & 0,0781 & 0,0825 & 0,8817 & $-0,0136$ & 0,3005 & 0,2649 \\
\hline INDF & $-0,0538$ & 0,0955 & 0,0782 & 0,8467 & $-0,0372$ & 0,2989 & 0,6914 \\
\hline KLBF & $-0,0658$ & 0,0762 & 0,0772 & 0,9473 & 0,0029 & 0,2797 & $-0,0433$ \\
\hline UNVR & $-0,0715$ & 0,0743 & 0,0780 & 0,9319 & $-0,0035$ & 0,2842 & 0,0486 \\
\hline
\end{tabular}

